\title{
Statistical characteristics of falling-film flows: A synergistic approach at the crossroads of direct numerical simulations and experiments
}

\author{
Alexandros Charogiannis, ${ }^{1}$ Fabian Denner, ${ }^{2}$ Berend G. M. van Wachem, ${ }^{2}$ Serafim Kalliadasis,${ }^{3}$ \\ and Christos N. Markides ${ }^{1, *}$ \\ ${ }^{1}$ Clean Energy Processes (CEP) Laboratory, Department of Chemical Engineering, Imperial College London, \\ London SW7 2AZ, United Kingdom \\ ${ }^{2}$ Department of Mechanical Engineering, Imperial College London, London SW7 2AZ, United Kingdom \\ ${ }^{3}$ Department of Chemical Engineering, Imperial College London, London SW7 2AZ, United Kingdom
}

(Received 22 February 2017; published 8 December 2017)

\begin{abstract}
We scrutinize the statistical characteristics of liquid films flowing over an inclined planar surface based on film height and velocity measurements that are recovered simultaneously by application of planar laser-induced fluorescence (PLIF) and particle tracking velocimetry (PTV), respectively. Our experiments are complemented by direct numerical simulations (DNSs) of liquid films simulated for different conditions so as to expand the parameter space of our investigation. Our statistical analysis builds upon a Reynolds-like decomposition of the time-varying flow rate that was presented in our previous research effort on falling films in [Charogiannis et al., Phys. Rev. Fluids 2, 014002 (2017)], and which reveals that the dimensionless ratio of the unsteady term to the mean flow rate increases linearly with the product of the coefficients of variation of the film height and bulk velocity, as well as with the ratio of the Nusselt height to the mean film height, both at the same upstream PLIF/PTV measurement location. Based on relations that are derived to describe these results, a methodology for predicting the mass-transfer capability (through the mean and standard deviation of the bulk flow speed) of these flows is developed in terms of the mean and standard deviation of the film thickness and the mean flow rate, which are considerably easier to obtain experimentally than velocity profiles. The errors associated with these predictions are estimated at $\approx 1.5 \%$ and $8 \%$ respectively in the experiments and at $<1 \%$ and $<2 \%$ respectively in the DNSs. Beyond the generation of these relations for the prediction of important film flow characteristics based on simple flow information, the data provided can be used to design improved heat- and mass-transfer equipment reactors or other process operation units which exploit film flows, but also to develop and validate multiphase flow models in other physical and technological settings.
\end{abstract}

DOI: 10.1103/PhysRevFluids.2.124002

\section{INTRODUCTION}

\section{A. Problem definition and motivation}

Liquid films play a prominent role in the design of effective means of heat and mass transfer in a wide range of industrial and engineering systems, such as wetted-wall absorbers, heat exchangers, film condensers and evaporators, reactors and microcooling schemes, to name but a few. The development of waves on the free surface of liquid films has been linked to appreciable heat- and mass-transfer enhancements [1-4] that are attributed to a rich variety of complex flow phenomena

\footnotetext{
*Corresponding author: c.markides@imperial.ac.uk
}

Published by the American Physical Society under the terms of the Creative Commons Attribution 4. 0 International license. Further distribution of this work must maintain attribution to the author $(s)$ and the published article's title, journal citation, and DOI. 
that underlie these flows. The pioneering work of Kapitza and Kapitza [5,6] inspired numerous research efforts on falling films trying to elucidate their complex underlying dynamics, generating an abundance of theoretical, numerical, and experimental scientific studies. Yet, several key aspects associated with the formation and development of hydrodynamic waves, and with the flow field underneath the wavy interface in particular, continue to elude us. This is evidenced by the limited number of experimental studies that have reported simultaneously conducted and spatiotemporally resolved film-thickness and velocity-field measurements, which are particularly challenging to recover, predominately owing to the restricted fluid domains and the intermittent nature of the wavy interface.

The research effort described here, therefore, aims primarily to identify possible links between the statistics of the film thickness and bulk velocity, specifically by combining simultaneous measurements of spatiotemporally resolved liquid film height and liquid-phase velocity in harmonically excited falling films, with state-of-the-art direct numerical simulations (DNSs). Beyond cross-validation of the two approaches, the latter are also used in order to expand the observations and findings to a wider range of flow conditions, allowing more generalized conclusions to be drawn.

\section{B. Background}

Gravity-driven, or "falling," liquid films are generally unstable to long-wavelength perturbations above a critical Reynolds number, $\operatorname{Re}_{\mathrm{c}}$ (see, for example, Ref. [7]), leading to the formation of freesurface instabilities. These instabilities evolve into highly asymmetric solitary waves with steep fronts preceded by capillary ripples via a secondary modulation instability [8-12], which sufficiently far from the inlet break down into continuously interacting three-dimensional (3-D) waves [8,9,11,13$18]$, and finally form rivulets at sufficiently long distances from the flow inlet $[16,17,19]$. By forcing the inlet flow rate at a prescribed frequency, the development of wave sequences which adopt this frequency can instead be triggered selectively. The interfacial characteristics of harmonically excited liquid films have been investigated analytically, numerically, and experimentally by many researchers [13,20-30], who have shown that depending on the excitation frequency, a range of different evolution scenarios can be realized. Our experiments and simulations employ low forcing-frequency excitation, following which the growth of solitary waves saturates within a few wavelengths from the inlet, while their shapes fluctuate modestly through interactions with their neighbors.

In the case of a one-dimensional (1-D), steady, fully developed, gravity-driven film flow, the formation of interfacial waves is suppressed (i.e., the film height remains constant), and the velocity profile across the film can be calculated from the Nusselt solution to the Navier-Stokes (NS) equations [31]. The Nusselt film height, $h_{\mathrm{N}}$, is expressed in Eq. (1) as a function of the fluid kinematic viscosity, $v_{\mathrm{f}}$, the gravitational acceleration, $g$, the inclination angle, $\beta$, and the (constant) volumetric flow rate per unit span of the flow, $Q$ :

$$
h_{\mathrm{N}}=\left(\frac{3 v_{\mathrm{f}} Q}{g \sin \beta}\right)^{1 / 3}=\left(\frac{3 v_{\mathrm{f}}^{2} \mathrm{Re}}{g \sin \beta}\right)^{1 / 3},
$$

where the flow Reynolds number here is expressed as a function of the volumetric flow rate per unit span of the flow, $Q$, and the kinematic viscosity of the liquid $\nu_{\mathrm{f}}$ :

$$
\operatorname{Re}=\frac{Q}{v_{\mathrm{f}}} .
$$

Here we adopt a definition for Re that is equivalent to the above but is more appropriate for unsteady wavy-film flows and is based on the time-mean volumetric flow rate per unit span, $\bar{Q}$, as measured by the flow meter installed in our setup.

Returning to the Nusselt flow, the liquid bulk velocity, also referred to as the Nusselt velocity, $U_{\mathrm{N}}$, can be obtained by integrating the axial-velocity profile $U_{\mathrm{x}}(y)$ in Eq. (3),

$$
U_{x}(y)=\frac{g \sin \beta}{v_{\mathrm{f}}} y\left(h-\frac{y}{2}\right),
$$


over the film height $h$, which gives

$$
U_{\mathrm{N}}=\frac{g \sin \beta h_{\mathrm{N}}^{2}}{3 v_{\mathrm{f}}} .
$$

The above simple expressions for $h_{\mathrm{N}}$ and $U_{\mathrm{N}}$ are often used as characteristic length and velocity scales in analytical and numerical studies (see, for example, Refs. [12,32,33], and in order to compare experimental results in a variety of real flows with the Nusselt (steady-flow) solution [34-37]. For example, in the limited number of experimental investigations of falling-film flows with measurements of axial-velocity profiles, these have been compared to profiles derived using Eq. (3), and experimentally recovered, local and instantaneous film-thickness data (see, for example, the comparisons by Moran et al. [36] for laminar films flowing down a $\beta=45^{\circ}$ incline over the range $\mathrm{Re}=11-220$ and those by Adomeit and Renz [37] for water films in the range $\mathrm{Re}=27-200$ ). In previous contributions by our group [38,39], we investigated the deviations between experimentally recovered and theoretical (Nusselt) velocity profiles systematically in harmonically excited liquidfilm flows pertaining to different flow $\operatorname{Re}(\operatorname{Re}=2.3-320)$, liquid Kapitza numbers, Ka $=85,350$, and 1800, and forcing frequencies, $f_{\mathrm{w}}=7$ and $10 \mathrm{~Hz}$; here, the fluid Ka depends on the liquid properties only and is defined as

$$
\mathrm{Ka}=\frac{\gamma_{\mathrm{f}}}{\rho_{\mathrm{f}} \nu_{\mathrm{f}}^{4 / 3} g \sin \beta^{1 / 3}},
$$

with $\gamma_{\mathrm{f}}$ representing the surface tension. In agreement with previous investigators, we reported significant excursions (by up to 100\%) of both bulk and interfacial velocities relative to the Nusselt solution at the wave crests, as well as a strong dependence of the magnitude of these deviations on the flow Re.

When comparing the mean film height, $\bar{h}$, with the Nusselt height, $h_{\mathrm{N}}$, evidence compiled by Tihon et al. using both experimental (over the range $\mathrm{Re}=10-100$ ) and numerical data [40,41] suggests that solitary waves reduce the wall shear stress in comparison to a flat interface. Hence, the fluid travels faster, on average, in a wavy-film flow compared to an equivalent flat-film flow of the same average flow rate. This finding implies that in order for the average flow rate to be conserved, the mean film height in a wavy-film flow must be lower than the equivalent Nusselt height $\left(\bar{h} \leqslant h_{\mathrm{N}}\right)$. Other experimental studies have, however, reported different trends. Moran et al. [36], for example, observed that $\bar{h} \geqslant h_{\mathrm{N}}$ throughout the range of flow conditions that were investigated in the study noted earlier, as did Takahama and Kato [42] over the range $\mathrm{Re}=200-997$. In contrast, the measurements provided by Lel et al. [43] over the range $\mathrm{Re}=2-700$, Zhou et al. [44] over the range $\mathrm{Re}=320-2060$, Ambrosini et al. [45] over the range $\mathrm{Re}=150-3300$, and Drosos et al. [46] over the range $\operatorname{Re}=60-360$ suggest that $\bar{h} \leqslant h_{\mathrm{N}}$ is obeyed below $\operatorname{Re} \approx 100-500$, and $\bar{h} \geqslant h_{\mathrm{N}}$ above that Re range. The transition from one behavior to another is shown to depend on the inclination angle, the measurement distance from the flow inlet, and properties of the employed fluid. It should finally be noted that both Karapantsios et al. [47] and Zadrazil et al. [48] report that $\bar{h} \geqslant h_{\mathrm{N}}$ for high-Re water flows $(\operatorname{Re}=509-13090$ and $\operatorname{Re}=306-1532$, respectively.)

In light of the large number of experimental studies in the literature that have reported such comparisons, two remarks can be made. First, irrespective of the trends observed in low-Re film flows, evidence exists that suggests a reversal of the $\bar{h} \leqslant h_{\mathrm{N}}$ relation at higher Re; this may potentially be linked to the onset of a transition to turbulence. Nevertheless, a possible flow mechanism responsible for this reversal has yet to be proposed. Second, despite evidence that the variation of $\bar{h} / h_{\mathrm{N}}$ (mean film height to Nusselt height ratio) is linked to the interface waviness and the associated bulk-velocity fluctuations, a relation describing this link has not been reported. Similarly, quantitative relationships that link the film thickness and velocity fluctuations are also lacking. Neither observation comes as a surprise, given that simultaneously acquired, space- and time-resolved film height and flow-field information from underneath the wavy interface would be required in order to develop such relations. In this contribution, we aim to address these deficits. 


\section{Aims and objectives}

The primary objectives of this work are to provide quantitative information on the statistics of unsteady film flows, specifically those relating to the dynamics of the interfacial waves in these flows, and to investigate their mass-transfer characteristics over the range of experimentally and numerically investigated conditions. The ultimate goal is to provide a set of relations linking the bulk-flow statistics, namely the mean bulk velocity and bulk-velocity standard deviation with the film-height statistics, and thus provide a tool for predicting the former using only knowledge of the latter.

Experimental data are recovered by the simultaneous application of planar laser-induced fluoresce (PLIF) and particle tracking velocimetry (PTV) [38], and are complemented by DNSs, for falling-film flows spanning the range $\mathrm{Re}=10-291$, liquids with $\mathrm{Ka}=85,350$, and 1800, and inlet (flow-rate) forcing frequencies of $f_{\mathrm{w}}=7$ and $10 \mathrm{~Hz}$. Additional DNSs at different upstream locations relative to the imaged (in the experiment) region of the flow are also performed, along with DNSs of film flows pertaining to different $\mathrm{Re}, \mathrm{Ka}$, and $\beta$ values, in order to expand the parameter space of our investigation. Following a brief introduction to the experimental techniques and numerical methodologies that are employed in our study, we present mean bulk velocity, bulk-velocity standard deviation, and bulk-velocity coefficient of variation results across the range of flow conditions that were investigated both experimentally and by DNSs. We then proceed to the main body of our analysis, which builds upon our previous research effort (see Ref. [39]), and specifically the Reynolds-like decomposition of the time-varying mean flow rate into steady and unsteady terms. In our previous paper, we showed that the former is represented by the product of the mean film height and mean bulk velocity, which varies linearly with the flow Re, and the latter by the covariance of the film-height and bulk-velocity fluctuations, which varies linearly with the film-height variance. These quantities were recovered experimentally, by generating film-height and bulk-velocity time series, and combining them into flow-rate time series. In the present study, we reproduce these results numerically, expand the analysis to include additional flow conditions, and study the evolution of the steady and unsteady terms as a function of distance from the flow inlet by DNSs. We then link the magnitude of these terms with the film-height and bulk-velocity statistics, as well as the magnitude of the $\bar{h} / h_{\mathrm{N}}$ ratio. Finally, we use these relations to devise an efficient and systematic methodology for predicting the bulk-velocity statistics, as well as the magnitude of the steady and unsteady terms, using only film-height information and the mean flow rate, which are considerably easier to obtain experimentally compared to two-dimensional (2-D) velocity distributions (these are necessary to recover the bulk-velocity statistics).

\section{EXPERIMENTAL METHODOLOGY}

\section{A. Experimental facility}

The experimental flow facility comprising the flow loop, test section, and optics is described in detail in previous publications (see Refs. $[4,38,39]$ ), and therefore only a brief summary is provided here for clarity and completeness. The test section over which liquid-film flows develop comprises a rectangular, $400 \times 290 \mathrm{~mm}$ glass sheet installed on an aluminium frame and inclined at $\beta=20^{\circ}$ to the horizontal [Fig. 1(a)]. The flow is dispensed uniformly along the span of the test section by a settling chamber featuring a knife edge, which is installed at the flow inlet. The liquid flow rate through the inlet (settling chamber, knife edge) and onto the test section planar substrate is pulsed. This is achieved, upstream of the chamber, by bypassing a portion of the flow through a throttle valve, which is rotated by a stepper motor in order to allow the accurate setting of the wave frequency. The amplitude of pulsation is determined by the fraction of the total flow that is directed through the valve. The time-varying flow rate is measured using an ultrasonic flowmeter installed upstream of the settling-chamber manifold.

Before proceeding with a presentation of the measurement techniques and numerical methodologies that we employ, it is essential to comment on the selection of the two $f_{\mathrm{w}}$ values that we impose. These were limited, at the upper bound, by our excitation setup which allows for disturbances of up 


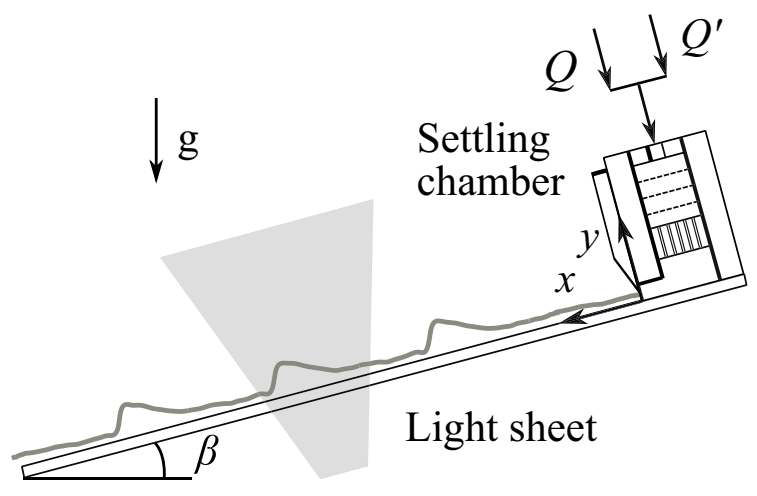

(a)

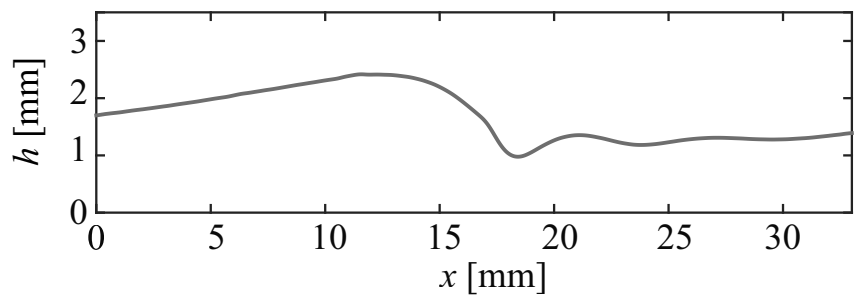

(b)

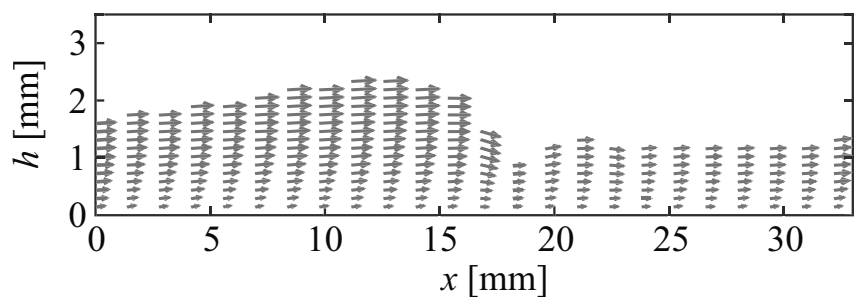

(c)

FIG. 1. Schematic of the experimental facility and sample wave- and phase-locked average, film height, and flow-field measurements by application of PLIF and PTV (reproduced from Figs. 1 and 4 in Ref [39]). (a) Test-section schematic showing the settling chamber and substrate, and the light-sheet orientation relative to the flow. (b) Wave- and phase-locked average film height near the crest of a solitary wave for a flow with $\mathrm{Re}=21, \mathrm{Ka}=85$, and $f_{\mathrm{w}}=7 \mathrm{~Hz}$. (c) Wave- and phase-locked average velocity field corresponding to panel (b).

to $\approx 11 \mathrm{~Hz}$ to be imposed by forcing the inlet flow rate periodically, and at the lower bound by the threshold frequency below which the regular wave pattern is destroyed by the emergence of parasitic waves (see, for example, Ref. [27]). Yet, as we showed in Ref. [39], the employment of $f_{\mathrm{w}}=7$ and $10 \mathrm{~Hz}$ often yields distinct wave topologies and film-height statistics. We would also like to emphasize that the selected frequencies do not coincide with the natural frequency (i.e., the most amplified mode), which depends on the fluid properties and can therefore vary with the liquid Ka. However, a comparison of the dynamics of films excited at their natural frequency to films excited at other frequencies falls beyond the scope of our current study, which rather focuses on relating the film-height to the bulk-velocity statistics over a large range of flow $\mathrm{Re}$ and liquid $\mathrm{Ka}$. 


\section{B. Optical diagnostic techniques}

In order to conduct PLIF and PTV measurements, the flow is seeded with rhodamine-B dye and glass hollow spheres (11 $\mu \mathrm{m}$ mean diameter) respectively. Excitation and imaging are both performed from the substrate side (underneath), in order to limit image distortions, by employment of a double-cavity frequency-doubled Nd:YAG laser operated at $100 \mathrm{~Hz}$ and a pair of LaVision VC-Imager Pro HS 500 CMOS cameras (one for collecting the rhodamine fluorescence and one for the particle scattering signal). The imaging planes of both cameras are mapped and corrected for perspective distortions using a calibration graticule immersed inside the employed liquid and a pinhole model available in LaVision's Davis software. The spatial resolution varies between 28.0 and $29.7 \mu \mathrm{m} /$ pixel depending on the experimental run. The imaged domain (region of interest) along the streamwise direction of the flow extends to approximately $33 \mathrm{~mm}$, and the downstream distance where measurements are collected corresponds to $0.256 \mathrm{~m}$, the furthermost downstream location that was optically accessible in our flow facility.

Both PLIF and particle images are subjected to perspective-distortion corrections and reflection removal. The two interfaces (solid-liquid and gas-liquid) are first identified in the PLIF images by means of dedicated MATLAB algorithms developed in house, and then mapped onto the particle images for masking. The particle images are used to calculate 2-D velocity-vector maps (PIV calculation) by means of a four-pass cross-correlation algorithm available in Davis, with a vector-to-vector spatial resolution of $\approx 220-240 \mu \mathrm{m}$. Individual particles are tracked (PTV calculation) by employment of the obtained PIV results as reference estimators of the velocity field.

\section{Error estimation}

A series of independent experiments were conducted for error estimation and technique validation purposes. Film-height measurements on flat films $(\mathrm{Ka}=14.1)$ were compared to Nusselt-height calculations, with a resulting average deviation of $\approx 20 \mu \mathrm{m}$. Based on this test, as well as other carefully designed validation experiments, the relative uncertainty associated with the instantaneous and local film-height measurements is estimated at $\leqslant 3 \%$.

PTV-derived interfacial and bulk-velocity measurements were also compared to Nusselt-solution results, with the corresponding mean deviations amounting to $3.2 \%$ for both velocity measures. Finally, based on flow rate comparisons between PLIF/PTV-derived and flow-meter data, a mean relative deviation of $1.6 \%$ was obtained over a total of six flat and nine harmonically excited flows. This value is smaller than the uncertainty associated with the flow-meter measurement (3\%), so the latter is used as an estimate of our mean flow-rate measurement error (for more details, the reader is referred to Refs. [38,39]).

\section{NUMERICAL METHODOLOGY}

Like with our experimental apparatus, the computational framework for interfacial flows has been developed in our previous works [49-53] and the reader is referred to these for technical details. Here we review the main ingredients of our framework, again for the purposes of clarity and completeness.

The incompressible flow of the immiscible two-phase system is governed by the momentum equations (using the Einstein notation),

$$
\rho\left(\frac{\partial u_{i}}{\partial t}+u_{j} \frac{\partial u_{i}}{\partial x_{j}}\right)=-\frac{\partial p}{\partial x_{i}}+\frac{\partial}{\partial x_{j}}\left[\mu\left(\frac{\partial u_{i}}{\partial x_{j}}+\frac{\partial u_{j}}{\partial x_{i}}\right)\right]+\rho g_{i}+f_{\sigma, i},
$$

and the continuity equation,

$$
\frac{\partial u_{i}}{\partial x_{i}}=0
$$


where $t$ represents time, $\boldsymbol{u}$ is the velocity, $p$ is the pressure, $\rho$ is the density, $\mu$ is the dynamic viscosity, and $\boldsymbol{f}_{\sigma}$ is the volumetric surface force acting at the liquid-gas interface.

The governing equations are solved using a general-purpose finite-volume framework for the simulation of interfacial flows [49], resolving all relevant length and time scales. The momentum equations, see Eq. (6), are discretized using a second-order backward Euler scheme for the transient term while the spatial terms are discretized using central differencing [49]. The continuity equation, see Eq. (7), is discretized using a specifically designed momentum-weighted interpolation method proposed by Ref. [49].

The volume-of-fluid (VOF) method [54] is adopted to capture the interface separating the gas and liquid phases. The local volume fraction of both phases is represented in each mesh cell by the color function $\phi$, with $\phi=0$ in the gas phase and $\phi=1$ in the liquid phase. The interface is located in mesh cells with $0<\phi<1$. The local density $\rho$ and viscosity $\mu$ are calculated by an arithmetic average based on the local color function $\phi$ [50]. The advection of $\phi$ is governed by the linear advection equation:

$$
\frac{\partial \phi}{\partial t}+u_{i} \frac{\partial \phi}{\partial x_{i}}=0
$$

based on the underlying flow with velocity $\boldsymbol{u}$, which is discretized using a compressive VOF methodology [51]. The surface force per unit volume is described by the continuum surface force (CSF) model [55] as

$$
f_{\gamma, i}=\sigma \kappa \frac{\partial \phi}{\partial x_{i}}
$$

where $\kappa$ is the interface curvature, assuming a constant surface tension coefficient $\gamma$ and neglecting mass transfer between the bulk phases. No convolution is applied to smooth the surface force [52].

The 3-D computational domain has dimensions $L_{x} \times L_{y} \times 0.1 h_{\mathrm{N}}$, with $L_{x}$ being case dependent and $L_{y}=4-6 h_{\mathrm{N}}$. The computational domain is represented by a Cartesian mesh with a minimum resolution of 10 cells per Nusselt film height $h_{\mathrm{N}}$. The numerical time step applied in the presented simulations satisfies a Courant number of $\mathrm{Co}=\Delta t_{u}|\boldsymbol{u}| / \Delta x \leqslant 0.25$, as well as the capillary time-step constraint [53]. A no-slip condition is enforced at the substrate and a free-slip boundary condition is applied at the top (gas-side) boundary. At the domain inlet the film height is constant, $h(x=0)=h_{\mathrm{N}}$, and a semiparabolic velocity profile is prescribed for the liquid phase, including a monochromatic forcing with frequency $f$ and amplitude $A$, given as

$$
u\left(x=0,0 \leqslant y \leqslant h_{\mathrm{N}}\right)=\frac{3}{2}[1+A \sin (2 \pi f t)]\left(\frac{2 y}{h_{\mathrm{N}}}-\frac{y^{2}}{h_{\mathrm{N}}^{2}}\right) U_{\mathrm{N}} .
$$

For the gas phase, a spatially invariant velocity is prescribed:

$$
u\left(x=0, h_{\mathrm{N}}<y \leqslant 4 h_{\mathrm{N}}\right)=\frac{3}{2}[1+A \sin (2 \pi f t)] U_{\mathrm{N}} .
$$

The domain outlet is modeled as an open boundary [25,33], which assures that the flow can leave the domain with minimal reflections. Initially, at time $t=0$, the velocity field is fully developed and the film is flat.

\section{FLOW STATISTICS}

In previous contributions, we compared wave crest and trough height, wave speed, interfacial velocity, and axial velocity profile data generated by the application of PLIF and PTV with data generated by DNSs of the same flows and at the same downstream location where experimental measurements were collected [39]. In the following sections we present and compare experimental 
TABLE I. Flow conditions and liquid properties for the falling-film flows examined in the present work. Measurements were collected at a distance $0.256 \mathrm{~m}$ downstream of the flow inlet. The substrate inclination angle was set to $\beta=20^{\circ}$ in all cases.

\begin{tabular}{lcccccc}
\hline \hline $\operatorname{Re}$ & $f_{\mathrm{w}}[\mathrm{Hz}]$ & $\mathrm{Ka}$ & $\nu_{\mathrm{f}}\left[10^{6} \mathrm{~m}^{2} \mathrm{~s}^{-1}\right]$ & $\rho_{\mathrm{f}}\left[\mathrm{kg} \mathrm{m}^{-3}\right]$ & $\gamma_{\mathrm{f}}\left[10^{3} \mathrm{~N} \mathrm{~m}^{-1}\right]$ & Method \\
\hline 11 & 7,10 & 85 & 15.6 & 1170 & 58.7 & Exp \& DNS \\
14 & 7,10 & 85 & 15.6 & 1170 & 58.7 & Exp \\
21 & 7,10 & 85 & 15.6 & 1170 & 58.7 & Exp \& DNS \\
25 & 7,10 & 85 & 15.6 & 1170 & 58.7 & Exp \& DNS \\
37 & 7,10 & 350 & 5.77 & 1113 & 59.7 & Exp \\
45 & 7,10 & 350 & 5.77 & 1113 & 59.7 & Exp \& DNS \\
54 & 7 & 350 & 5.77 & 1113 & 59.7 & Exp \\
65 & 7,10 & 350 & 5.77 & 1113 & 59.7 & Exp \& DNS \\
113 & 7,10 & 1800 & 1.71 & 1170 & 58.1 & Exp \\
152 & 7,10 & 1800 & 1.71 & 1170 & 58.1 & Exp \\
195 & 7,10 & 1800 & 1.71 & 1170 & 58.1 & Exp \\
238 & 7,10 & 1800 & 1.71 & 1170 & 58.1 & Exp \\
291 & 7,10 & 1800 & 1.71 & 1170 & 58.1 & Exp \\
\hline \hline
\end{tabular}

("Exp") and DNS results on film waviness, based on two quantitative measures of this film characteristic: (i) the standard deviations of the film height and bulk velocity, $\sigma_{\mathrm{h}}$ and $\sigma_{\mathrm{U}}$, respectively; and (ii) the film-height and bulk-velocity coefficients of variation (standard deviations normalized by the respective means), $\sigma_{\mathrm{h}} / \bar{h}$ and $\sigma_{\mathrm{U}} / \bar{U}$, respectively. To the best of our knowledge, such comparisons have not been pursued previously in the literature. The flow Re, fluid (liquid) Ka, and forcing frequency, $f_{\mathrm{w}}$, of the investigated flow conditions in the experiments and DNSs are given in Table I. The flows considered comprise three aqueous-glycerol solutions $(\mathrm{Ka}=85,350$, and 1800), $\mathrm{Re}$ spanning the ranges $\mathrm{Re}=10-25,37-$ and 112- respectively, and forcing frequencies $f_{\mathrm{w}}=7$ and $10 \mathrm{~Hz}$. DNS data are provided for three Re per liquid solution for flows pertaining to liquids with $\mathrm{Ka}=85$ and 350 .

In the experiments, film-height and bulk-velocity statistics were calculated from time series such as those presented in Fig. 2 (data corresponding to a $\approx 0.8$-s recording period), for a flow with $\mathrm{Ka}=350, \mathrm{Re}=65$, and $f_{\mathrm{w}}=10 \mathrm{~Hz}$. These were, in turn, generated by space averaging the measured film heights and bulk velocities locally over a 100 -pixel $(\approx 2.8-\mathrm{mm})$ flow region on a per-image basis, with each PLIF/PTV image pair providing a pair of film height and bulk velocity data points. For film flows excited at $f_{\mathrm{w}}=7 \mathrm{~Hz}$, a total of 700 images were recorded per flow condition, while for film flows excited at $f_{\mathrm{w}}=10 \mathrm{~Hz}$, a total of 500 images were recorded instead, yielding in both cases temporal records that comprise $\approx 50$ waves. Film-height standard deviations were also calculated, for a wide range of flow conditions, using our entire data records (i.e., all measurement points in each image and all available images), as well as time series that were compiled using data that were extracted locally, but from nonsuccessive frames. The standard deviations that were obtained from the different data samples did not vary by more $\approx 3 \%$ for the same flow condition.

The mean film heights, $\bar{h}$, and mean bulk velocities, $\bar{U}$, were calculated using Eqs. (12) and (13), while the film-height and bulk-velocity standard deviations, $\sigma_{\mathrm{h}}$ and $\sigma_{\mathrm{U}}$, were calculated using Eqs. (14) and (15), respectively, with $N=700$ when $f_{\mathrm{w}}=7 \mathrm{~Hz}$ and $N=500$ when $f_{\mathrm{w}}=10 \mathrm{~Hz}$. The mean values are illustrated as continuous lines along the time-varying signals in Fig. 2, while the standard deviations are represented by dashed lines of magnitude equal to $\bar{h} \pm \sigma_{\mathrm{h}}$ and $\bar{U} \pm \sigma_{\mathrm{U}}$, respectively. Also drawn about the mean values of the film height and bulk velocity are the film-height and bulk-velocity fluctuations (blue stems), which are calculated according to $h_{i}^{\prime}=h_{i}-\bar{h}$ and 


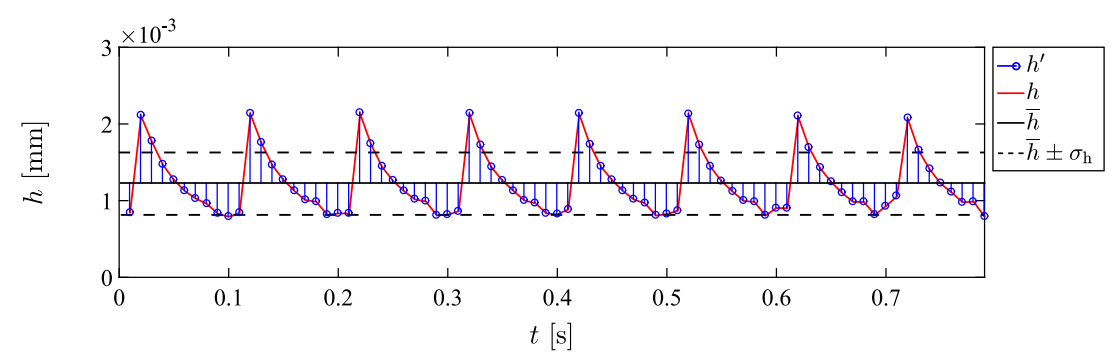

(a)

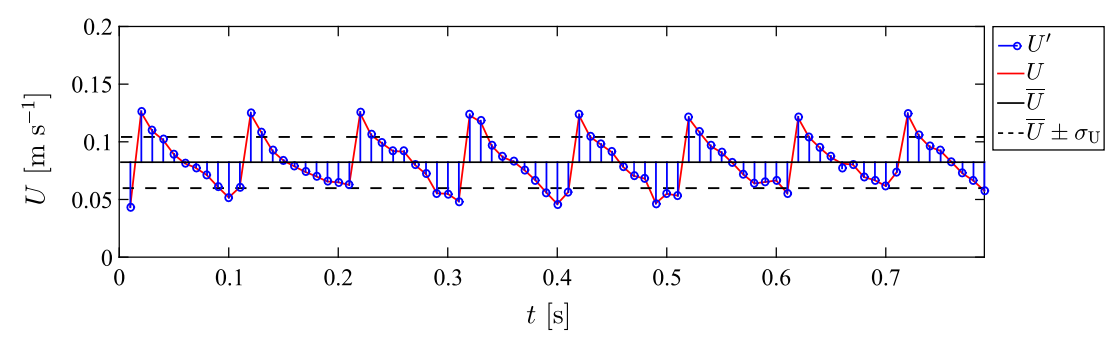

(b)

FIG. 2. (a) Film-height and (b) bulk-velocity time series plotted over a $\approx 0.8$-s recording-period for a flow with $\mathrm{Ka}=350, \mathrm{Re}=65$, and $f_{\mathrm{w}}=10 \mathrm{~Hz}$. Also shown are the film-height and bulk-velocity fluctuations, $h^{\prime}$ and $U^{\prime}$, respectively, the mean film height and bulk velocity, $\bar{h}$ and $\bar{U}$, respectively, and the film-height and bulk-velocity standard deviations, $\sigma_{\mathrm{h}}$ and $\sigma_{\mathrm{U}}$, respectively.

$U_{i}^{\prime}=U_{i}-\bar{U}$, respectively, at every point, $i$, along the time series:

$$
\begin{aligned}
\bar{h} & =\frac{1}{\mathrm{~N}} \sum_{i=1}^{\mathrm{N}} h_{i}, \\
\bar{U} & =\frac{1}{\mathrm{~N}} \sum_{i=1}^{\mathrm{N}} U_{i}, \\
\sigma_{\mathrm{h}} & =\sqrt{\frac{1}{\mathrm{~N}} \sum_{i=1}^{\mathrm{N}}\left(h_{i}-\bar{h}\right)^{2},} \\
\sigma_{\mathrm{U}} & =\sqrt{\frac{1}{\mathrm{~N}} \sum_{i=1}^{\mathrm{N}}\left(U_{i}-\bar{U}\right)^{2} .}
\end{aligned}
$$

The procedure that we followed to extract the film height and velocity data from the processed PLIF and PTV images is described in greater detail in Ref. [39], where local film-height and bulk-velocity measurements were combined in order to generate flow-rate time series. The latter were used to access the validity and accuracy of the combined PLIF/PTV measurement technique by comparing the obtained mean flow-rate data with flow-meter measurements, and also to link, via a simple linear relation that included the mean flow rate and the wave speed, the local and instantaneous flow rate to the fluctuation of the local and instantaneous film height. In the DNSs, the film-height and bulk-velocity statistics were calculated from data that were generated at the same downstream distance, $x=0.256 \mathrm{~m}$, as the experimental data, as well as other locations upstream of the former, over one spatial period (wavelength). 


\section{A. Film-height statistics}

Film-height standard deviation data relating to the examined film-flow conditions have been presented in a previous publication [39], and thus, we only reiterate here any results that are essential to the upcoming analysis. With increasing $\mathrm{Re}, \sigma_{\mathrm{h}}$ increases; however, at the highest $\operatorname{Re}(\operatorname{Re}=65)$ with $\mathrm{Ka}=350$ and $f_{\mathrm{w}}=10 \mathrm{~Hz}$, there is some evidence of saturation. Despite the fact that these trends are identified in both experimental and DNS data, the experimental $\sigma_{\mathrm{h}}$ values are higher than the DNS values (in the flow cases with $\mathrm{Ka}=85$, the average deviation between experiments and DNSs does not exceed 5\%, while in those with $\mathrm{Ka}=350$, the average deviation corresponds to approximately 13\%). The observed deviations, which increase with the flow Re and liquid Ka, can be directly attributed to the typically larger wave amplitudes, $\Delta h=h_{\max }-h_{\min }$, as well as the more pronounced film-height fluctuations in the capillary-wave regions observed in the experiments. These, in turn, can be linked to the parabolic crestline shapes of the nominally 2-D waves, which trigger a modest increase of the wave amplitudes along the test-section centerline, depending on the liquid $\mathrm{Ka}$ and channel width $w$ (see, for example, Ref. [56]). It should also be noted at this stage that the finite width of our test section and the presence of side walls in the experiment (unlike in the DNS) are not expected to markedly suppress the primary instability. Vlachogiannis et al. [57] and Leontidis et al. [58] have shown that at low inclination angles the dependence of the stability characteristics of film flows on the channel width is particularly prominent. However, for the range of flow $\operatorname{Re}$, the inclination angle $\beta$, and the channel width $w$ employed in our experimental campaign, the flow behavior should mimic closely that of an infinitely wide channel.

For the $\mathrm{Ka}=1800$ flows, on the other hand, $\sigma_{\mathrm{h}}$ decreases slightly with increasing Re over the range $\mathrm{Re}=112-291$. The ratio $\sigma_{\mathrm{h}} / \bar{h}$ decreases monotonically throughout the range of examined flow $\mathrm{Re}$ for both the $\mathrm{Ka}=350$ and $\mathrm{Ka}=1800$ data sets, whereas it first increases and subsequently saturates in the case of the lower $\mathrm{Ka}=85$ flows. Among the presented data, $\sigma_{\mathrm{h}} / \bar{h}$ peaks at a maximal value of 0.43 in the case of the $\mathrm{Re}=37, \mathrm{Ka}=350$, and $f_{\mathrm{w}}=7 \mathrm{~Hz}$ flow.

\section{B. Bulk-velocity statistics}

Much smaller deviations (of the order of the experimental errors associated with the film height, bulk velocity, density, viscosity, surface tension, and flow rate measurements and any numerical errors) are observed between our experimental and DNS bulk-velocity results depicted in Fig. 3. Furthermore, the trends observed for $\sigma_{\mathrm{U}}$ data, and more so the $\sigma_{\mathrm{U}} / \bar{U}$ data, deviate significantly from those discussed earlier for $\sigma_{\mathrm{h}}$ and $\sigma_{\mathrm{h}} / \bar{h}$, except for flows with $\mathrm{Ka}=85$ that show similar trends. Specifically, $\sigma_{\mathrm{U}}$ increases over all Re for the $\mathrm{Ka}=350$ flows, and is almost invariant to $\mathrm{Re}$ for $\mathrm{Ka}=1800$, whereas $\sigma_{\mathrm{U}} / \bar{U}$ falls rapidly with increasing Re in both cases over the same range of conditions. This is in contrast to the flows with $\mathrm{Ka}=85$, in which $\sigma_{\mathrm{U}} / \bar{U}$ increases with increasing Re before saturating (similarly to $\sigma_{\mathrm{h}} / \bar{h}$ ). Interestingly, the bulk-velocity coefficient of variation peaks at a near-identical value as the film-height coefficient of variation, i.e., $\sigma_{\mathrm{U}} / \bar{U} \approx 0.45$. Owing to the lack of such bulk-velocity information in the literature, no comparisons between our results and those of other researchers were pursued.

The different trends between $\sigma_{\mathrm{U}} / \bar{U}$ and Re on one hand, and $\sigma_{\mathrm{h}} / \bar{h}$ and Re on the other, can largely be attributed to the significantly stronger variation of $\bar{U}$ compared to $\bar{h}$. Based on the Nusselt solution to the NS equation, the bulk velocity scales with $h^{2}$; even though we have shown in Refs. [38,39] that this relation under- or overpredicts the true flow velocity depending on the film topology, $\bar{U}$ increases (incrementally) more with the Re than $\bar{h}$ does. For example, for the examined flows with $\mathrm{Ka}=350$ and $f_{\mathrm{w}}=7$ and $10 \mathrm{~Hz}, \bar{h}$ increases by $\approx 30 \%$ between $\mathrm{Re}=37$ and 76 for either wave frequency, while $\bar{U}$ increases by $\approx 70 \%$ and $\approx 55 \%$, respectively. Thus, as the increase of $\bar{U}$ is not compensated by an increase of similar extent in the $\sigma_{\mathrm{U}}$ data, $\sigma_{\mathrm{U}} / \bar{U}$ falls, at least for the flows with $\mathrm{Ka}=350$ and $\mathrm{Ka}=1800$. Regarding the $\mathrm{Ka}=85$ films, we observe that $\sigma_{\mathrm{U}}$ doubles over the examined Re range when $f_{\mathrm{w}}=7 \mathrm{~Hz}$, and triples when $f_{\mathrm{w}}=10 \mathrm{~Hz}$, while $\bar{U}$ increases, once more, by $\approx 70 \%$. The increase in $\sigma_{\mathrm{U}} / \bar{U}$ with the flow Re, in this case, is attributed to the growth 


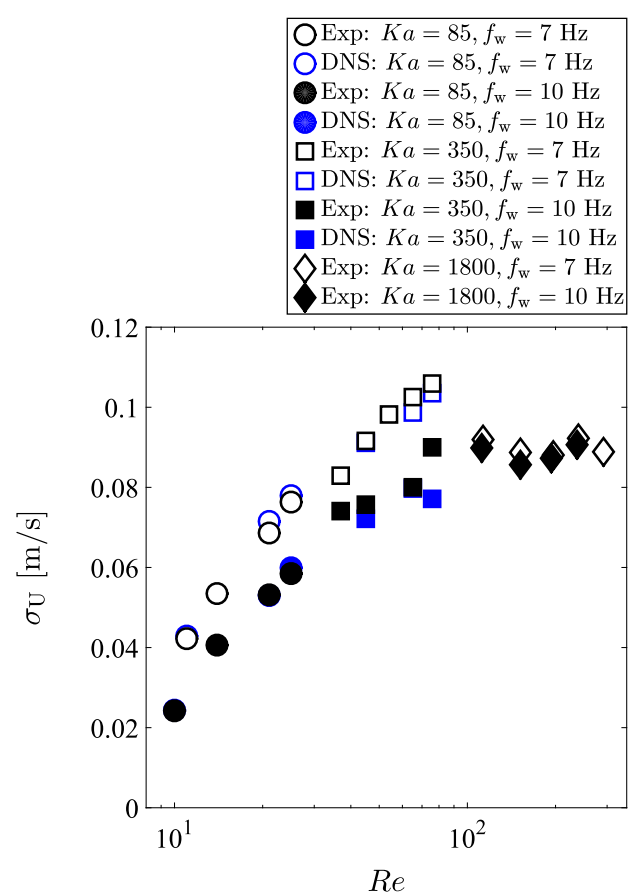

(a)

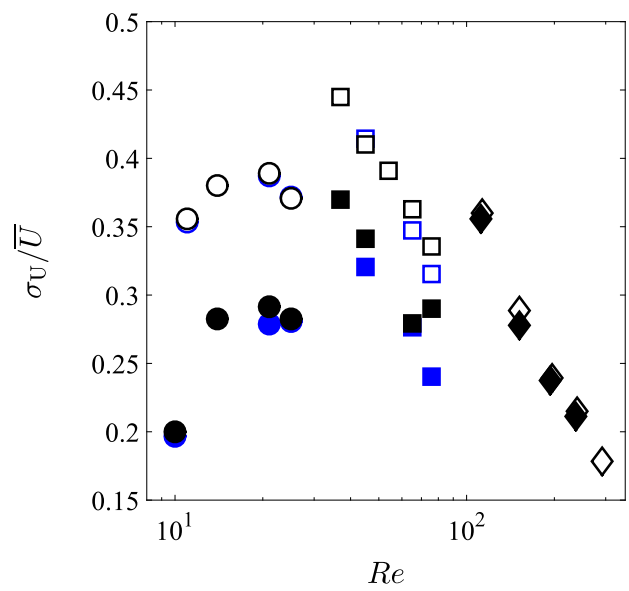

(b)

FIG. 3. Bulk-velocity statistics obtained by application of PTV and DNSs of the same flows. (a) Bulkvelocity standard deviation, $\sigma_{\mathrm{U}}$, plotted against the flow Re, for the flow conditions provided in Table I. (b) Coefficient of variation of the bulk-velocity, $\sigma_{U} / \bar{U}$, plotted against the flow Re for the same flow conditions.

of high-amplitude solitary waves over the examined Re range (see Ref. [39]), as evidenced by the significant increase in $\sigma_{\mathrm{h}}$ (by $\approx 70 \%$ when $f_{\mathrm{w}}=7 \mathrm{~Hz}$ and $\approx 140 \%$ when $f_{\mathrm{w}}=10 \mathrm{~Hz}$ between $\operatorname{Re} \approx 10-25$ ).

Figure 4 is a plot of $\sigma_{\mathrm{h}} / \bar{h}$ against $\sigma_{\mathrm{U}} / \bar{U}$, and reveals near-linear relationships with some indication that data generated for $f_{\mathrm{w}}=7$ and $10 \mathrm{~Hz}$ pertaining to the same Ka may collapse over the range of investigated Re. This collapse is strongest for the data obtained from the Ka = 1800 films: With increasing $\mathrm{Re}$, the film-height coefficient of variation varies almost linearly with the bulk-velocity coefficient of variation. Despite the trends observed among flows forced at the same frequency and pertaining to the same liquid Ka, a clear relationship between $\sigma_{\mathrm{h}} / \bar{h}$ against $\sigma_{\mathrm{U}} / \bar{U}$ cannot be derived based on the available experimental and numerical data. The link between the interface $\left(\sigma_{\mathrm{h}} / \bar{h}\right)$ and the flow-field unsteadiness $\left(\sigma_{\mathrm{U}} / \bar{U}\right)$ will, however, be considered in the next section.

\section{FLOW UNSTEADINESS}

\section{A. Reynolds decomposition of the time-varying flow rate}

In our previous contribution in Ref. [39], we examined the impact of flow unsteadiness on the mass-transfer capacity of harmonically excited falling films by a Reynolds-like decomposition of the time-varying flow rate per unit span of the flow into mean, $\bar{Q}$, and fluctuating, $Q^{\prime}$, components:

$$
Q=\bar{Q}+Q^{\prime}=U h .
$$

In particular, we showed that upon decomposing and averaging the time-varying flow rate per unit span, $Q$, the average flow rate, $\bar{Q}$, can be described by the sum of (i) the product of the mean bulk velocity, $\bar{U}$, and mean the film height $\bar{h}$ and (ii) the covariance of the film-height and bulk-velocity 


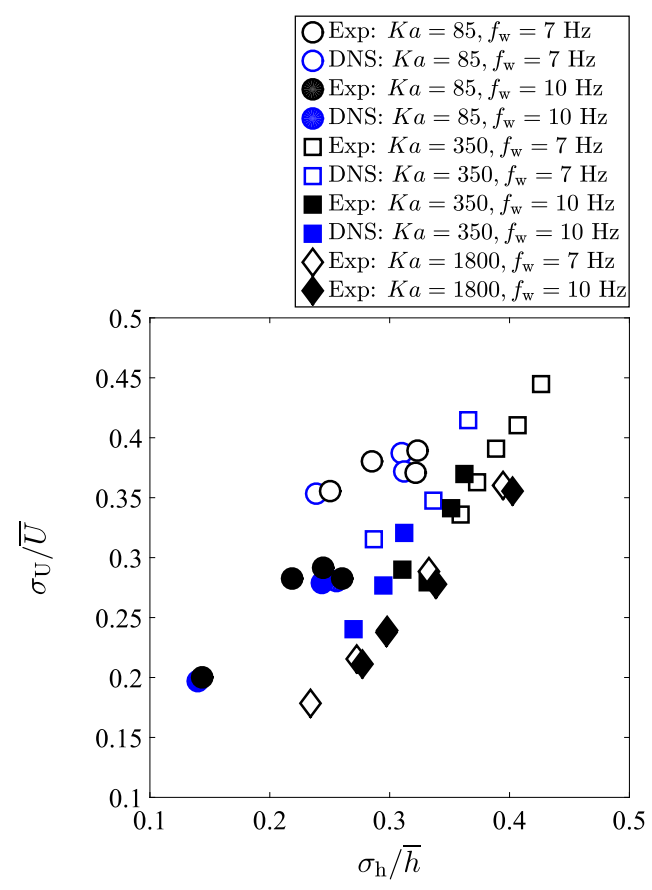

FIG. 4. Film-height coefficient of variation, $\sigma_{\mathrm{h}} / \bar{h}$, plotted against the bulk-velocity coefficient of variation, $\sigma_{\mathrm{U}} / \bar{U}$, over the range of flow conditions presented in Table $\mathrm{I}$.

fluctuations, $h^{\prime}$ and $U^{\prime}$, respectively, that represents the nonlinear coupling between the local and instantaneous film height $h$ and the local and instantaneous bulk velocity $U$. We refer to the first term of Eq. (17) as the steady term and to the second term as the unsteady term:

$$
\bar{Q}=\bar{U} \bar{h}+\overline{U^{\prime} h^{\prime}} .
$$

Over the range of examined flow conditions (Table I), the steady terms were found to vary linearly with Re, with gradients that were closely approximated by the fluid kinematic viscosity for each $\mathrm{Ka}$ data set, and the unsteady terms were shown to vary linearly with the film-height variance, $\sigma_{\mathrm{h}}^{2}$, effectively a measure of the film waviness.

Here we revisit these experimental results by (i) complementing them with DNSs, including simulations of water flows with $\mathrm{Ka}=4363, \beta=45^{\circ}, \mathrm{Re}=40,60,80,100$, and 120 , and $f_{\mathrm{w}}=$ $20 \mathrm{~Hz}$, and (ii) studying the evolution of the relative magnitudes of these terms as a function of distance from the flow inlet using DNSs. The latter will be used to enable a link between the flow statistics discussed in the pervious section and the flow unsteadiness, expressed as the ratio of the unsteady term to the mean flow rate, $\overline{U^{\prime} h^{\prime}} / \bar{Q}$.

The relation obtained between the steady terms and the flow $R e$ is presented in Fig. 5. We emphasize here that Re is based on the time-mean flow rate measured upstream of the inlet, $\bar{Q}$, and not the steady-term product $\bar{U} \bar{h}$ [the difference between the two was discussed above in relation to Eq. (17)]. With increasing Ka, the deviations between the gradients of linear fits to the data and the relation based on the fluid kinematic viscosities increase from $7 \%(\mathrm{Ka}=85)$ to $8 \%(\mathrm{Ka}=350), 9 \%$ $(\mathrm{Ka}=1800)$, and $11 \%(\mathrm{Ka}=4363)$.

The unsteady-term data are shown in Fig. 6. Even though experiments and DNSs display the same linear trends, the experimentally recovered $\sigma_{\mathrm{h}}^{2}$ and $\overline{U^{\prime} h^{\prime}} / \bar{Q}$ values are higher than their DNS counterparts for the same flow conditions. Based on our previous discussion regarding the subtle yet consistent underprediction of the film-height and bulk-velocity standard deviations by the DNSs, 


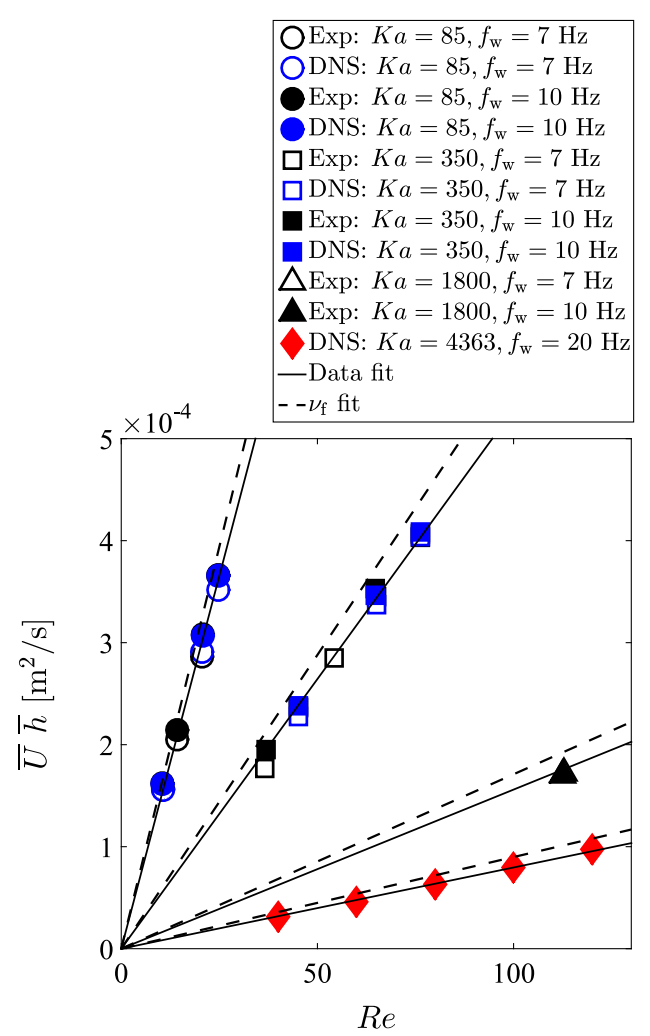

(a)

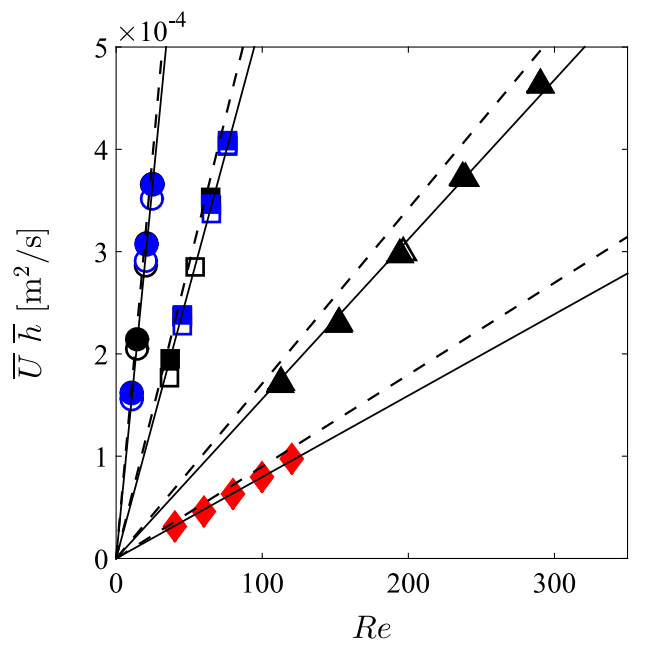

(b)

FIG. 5. Steady terms compiled using both experimental and numerical data for flows with $\mathrm{Ka}=85,350$, 1800, and 4363, plotted against the flow Re (based on the results presented in Fig. 13 in Ref [39].). Fits to the four Ka data sets are provided alongside plots of the mean flow rate against the flow Re, referred to in the legend as " $v_{\mathrm{f}}$ fit."

this result is unsurprising; the comparatively stronger variations of the interface height and bulk velocity are also reflected in the larger film-height and bulk-velocity fluctuation amplitudes. The linear relation between the unsteady terms and the film-height variance can be attributed to the high degree of correlation between $h$ and $U$. In the next section, we discuss the growth of the unsteady terms with increasing distance from the flow inlet, and therefore with increasing wave amplitude, and provide further insight regarding the coupling between the film-height and bulk-velocity fluctuations. It should, however, be noted that strong evidence in support of this thesis can already be found in the linear relation that we reported in Ref. [1], between the local and instantaneous flow rate and the local and instantaneous film height, based on direct experimental measurements as well as DNSs of the same flows. This result suggests that for each film height, the flow rate (in the axial direction of the flow) is fixed irrespective of the local film topology and according to a simple relation that comprises the wave speed, the mean flow rate, and the mean film height. The local and instantaneous flow rate is given by the product of the local and instantaneous film height and bulk velocity, and thus, the variation of the bulk velocity about the mean value (bulk-velocity fluctuation, $U^{\prime}$ ) is expected to track the film-height fluctuations, $h^{\prime}$, a statistical measure of which is the film-height standard deviation, $\sigma_{\mathrm{h}}$.

\section{B. Development of flow unsteadiness}

The observation of nonzero $\overline{U^{\prime} h^{\prime}}$ values in both experiments and DNSs is associated with flow unsteadiness at the downstream location where measurements were collected. In the case of a steady 


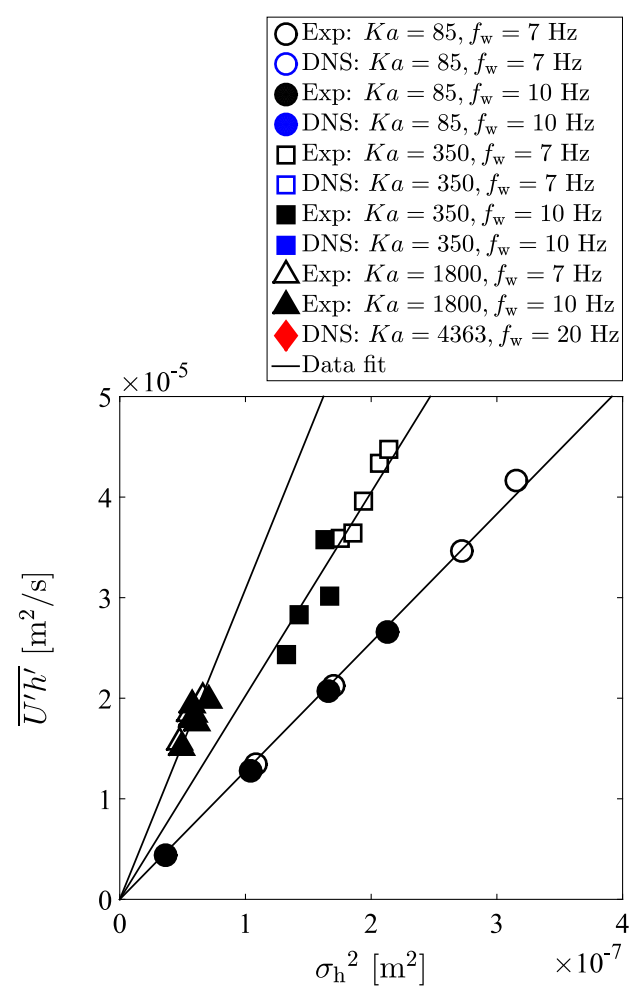

(a)

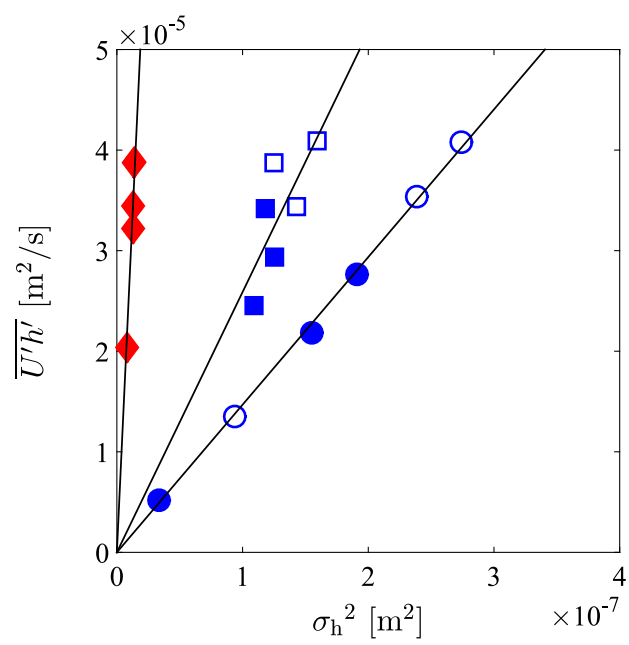

(b)

FIG. 6. Unsteady terms compiled using both experimental and numerical data for flows with $\mathrm{Ka}=85$, 350,1800 , and 4363, plotted against the film-height variance $\left(\sigma_{\mathrm{h}}^{2}\right)$ (based on the results presented in Fig. 14 in Ref. [39]). Linear fits to the four Ka data sets are also provided: (a) Experimental results. (b) Numerical results.

flow, for example, the flow immediately downstream of the inlet, both $U^{\prime}$ and $h^{\prime}$ are equal to zero and the mean flow rate can be described by

$$
\bar{Q}=\bar{U} \bar{h}=U_{\mathrm{N}} h_{\mathrm{N}} .
$$

Once free-surface instabilities emerge downstream of this short-lived flat-film region, they grow and eventually evolve into the 2-D solitary wave trains that are investigated here, as well in as our earlier studies $[4,33,38,39]$.

The development of interfacial waves is demonstrated in Fig. 7 by DNS of a film flow with $\mathrm{Ka}=350, \mathrm{Re}=45$, and $f_{\mathrm{w}}=7 \mathrm{~Hz}$, at distances $x=0,0.056,0.106$, and $0.256 \mathrm{~m}$ (the measurement location in the experiments) downstream of the flow inlet. In greater detail, the film height is plotted over one spatial period (i.e., one wavelength), showing the growth of the solitary-wave amplitude with increasing distance from the inlet which governs, along with the wavelength, the statistical quantities $\sigma_{\mathrm{h}} / \bar{h}$ and $\sigma_{\mathrm{U}} / \bar{U}$ of the flow. Based on this plot, we conjecture that the magnitude of the steady term will fall with increasing $x$ until wave growth saturates, while the magnitude of the unsteady term will increase. This prediction is examined in Fig. 8 over all flow conditions with $\mathrm{Ka}=85$ and 350 (see Table I) that were examined numerically. As expected, $\overline{U^{\prime} h^{\prime}} / \bar{Q} \approx 0$ and $\bar{U} \bar{h} / \bar{Q} \approx 1$ near the inlet, downstream of which $\bar{U} \bar{h} / \bar{Q}$ drops while $\overline{U^{\prime} h^{\prime}} / \bar{Q}$ increases. The highest value of the normalized unsteady term over the range of investigated flow conditions is $\approx 0.15$. Another interesting observation that relates to the results of Fig. 8 is that for certain flow conditions, for example, for the flow with $\mathrm{Ka}=350, \mathrm{Re}=45$, and $f_{\mathrm{w}}=10 \mathrm{~Hz}$, no saturation in the fall of $\bar{h} \bar{U} / \bar{Q}$ with $x$, or the growth of $\overline{U^{\prime} h^{\prime}} / \bar{Q}$ with $x$ is observed, while in others, such as the 


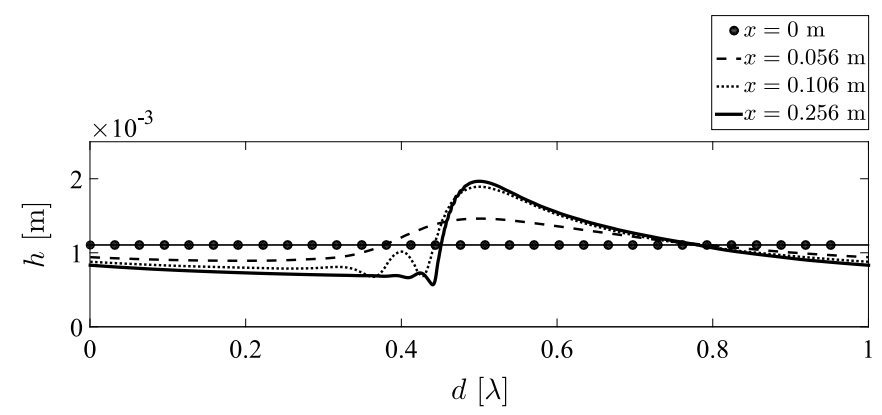

FIG. 7. Film height plotted over one spatial period (wavelength) for a flow with $\mathrm{Ka}=350, \mathrm{Re}=45$, and $f_{\mathrm{w}}=7 \mathrm{~Hz}$. The DNS results correspond to distances $x=0,56,106$, and $256 \mathrm{~mm}$ downstream of the flow inlet. The latter coincides with the location where PLIF/PTV data were collected.

$\mathrm{Ka}=85, \operatorname{Re}=10$, and $f_{\mathrm{w}}=10 \mathrm{~Hz}$ flow, both seem to level off with increasing $x$. If the magnitude of the steady and unsteady terms is used to gauge whether the growth of the examined 2-D waves has saturated (as the amplitude of the solitary waves increases the film-height and bulk-velocity fluctuations also increase), then the plotted data suggest that in some cases, these waves are still growing. The experimental and numerical results that we show later on (see, for example, Figs. 9 and 10), however, indicate that the relations we propose (linking the variation of the steady and unsteady terms to the coefficients of variation of the film height and the bulk velocity), are not

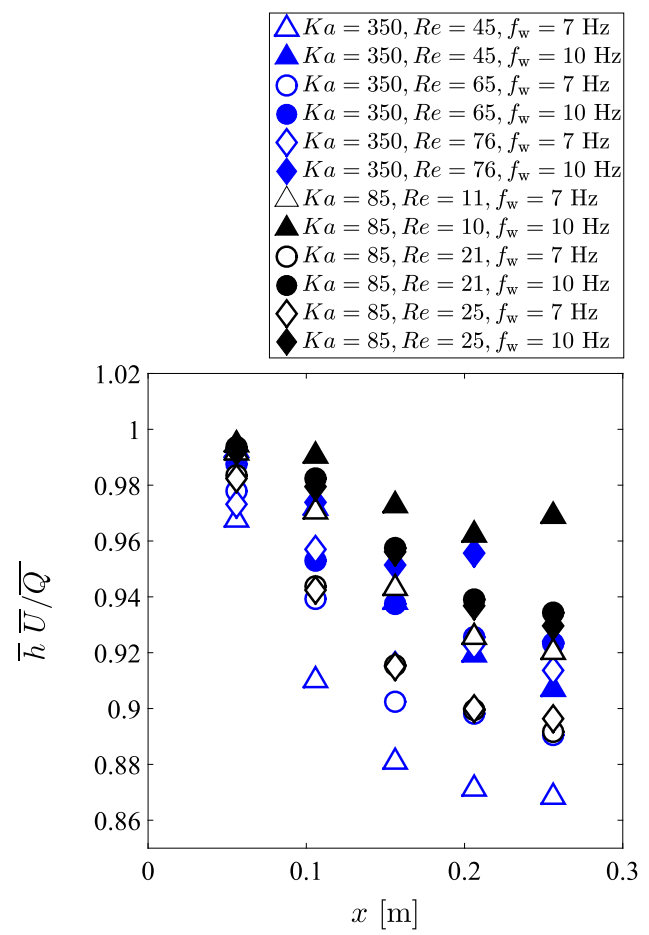

(a)

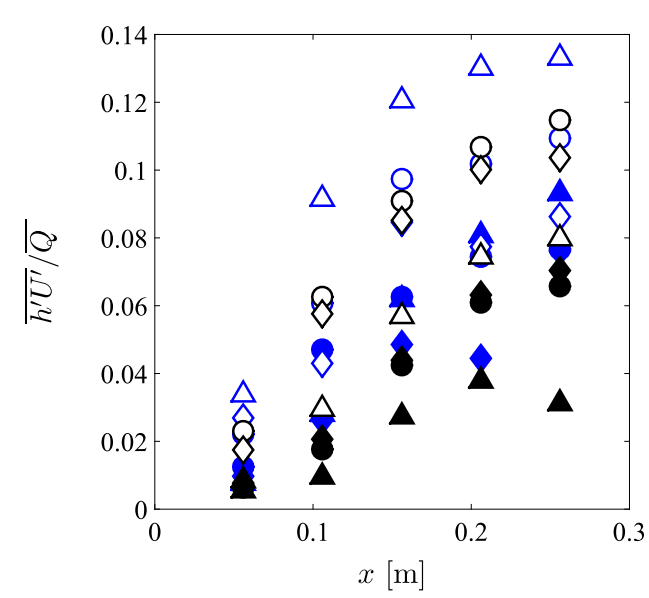

(b)

FIG. 8. Normalized steady, $\bar{U} \bar{h} / \bar{Q}$, and unsteady terms, $\overline{U^{\prime} h^{\prime}} / \bar{Q}$, plotted against the downstream distance from the inlet $(x=0.056,0.106,0.156,0.206,0.256 \mathrm{~m})$, for all flow conditions that were investigated numerically from Table I. 


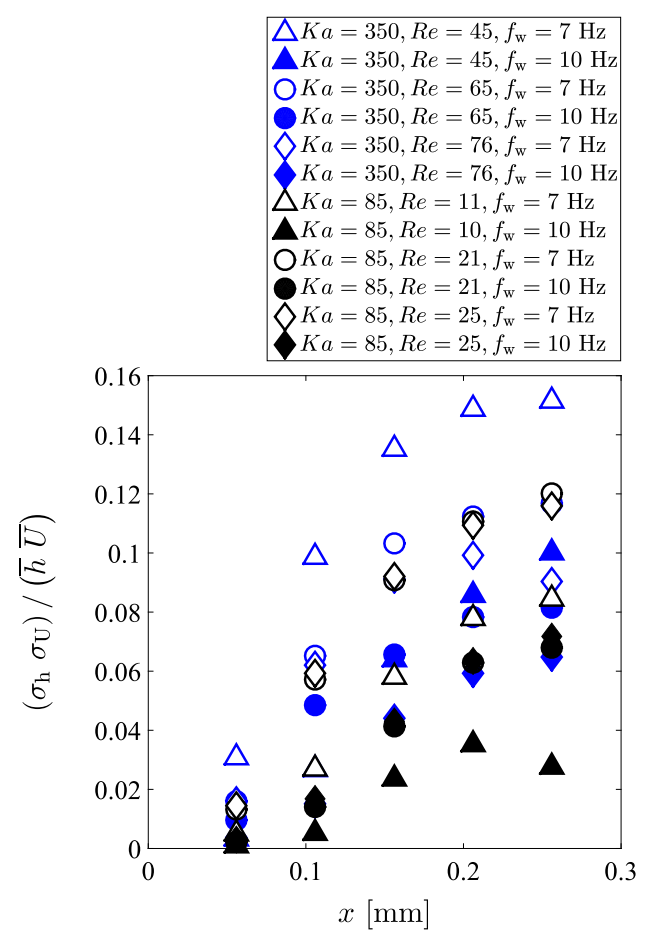

(a)

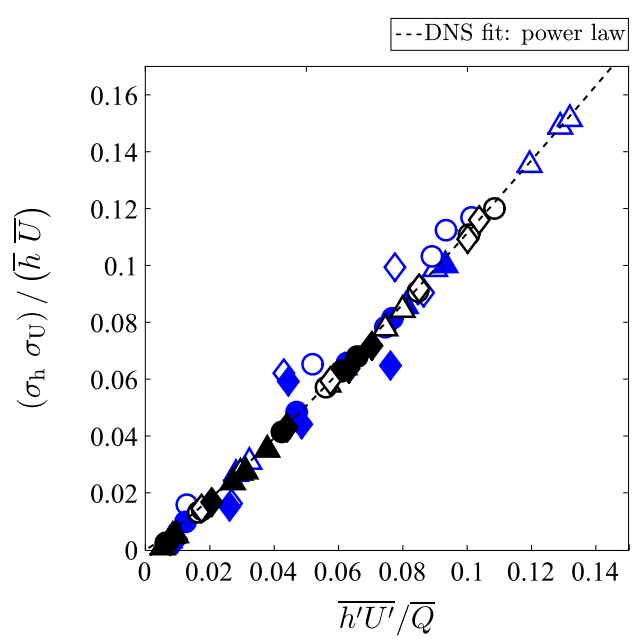

(b)

FIG. 9. Product of the coefficients of variation of the film height and bulk velocity, $\sigma_{\mathrm{h}} \sigma_{\mathrm{U}} /(\bar{h} \bar{U})$, against the downstream distance from the inlet $x=0.056,0.106,0.156,0.206$, and $0.256 \mathrm{~m}$, and of the normalized unsteady term, $\overline{U^{\prime} h^{\prime}} / \bar{Q}$, for all flow conditions from Table I that were simulated numerically.

contingent upon the saturation of the wave growth - but are instead applicable to any stage of the 2-D wave development, provided that no redistribution of liquid in the transverse direction of the flow (corresponding to the 2-D to 3-D wave transition) takes place at the examined region of the flow. This was confirmed in our experiments by comparing mean flow-rate measurements that were recovered by simultaneous application of PLIF/PTV, with independent ultrasonic flow-meter measurements across the range of investigated film-flow conditions [39] - but would not necessarily be the case if the film height was transversely modulated. Moreover, our mean film-height and bulk-velocity measurements, as well as our local film-height and velocity measurements that were generated along the investigated wave topologies, match the 2-D DNSs remarkably well throughout the range of examined film-flow conditions.

A plot of $\sigma_{\mathrm{h}} \sigma_{\mathrm{U}} /(\bar{h} \bar{U})$, i.e., the product of the coefficients of variation of the film height and bulk velocity, against $x$, the distance from the inlet, is provided in Fig. 9. The same quantity, $\sigma_{\mathrm{h}} \sigma_{\mathrm{U}} /(\bar{h} \bar{U})$, is also plotted against the unsteady term normalized by the mean flow rate, $\overline{U^{\prime} h^{\prime}} / \bar{Q}$, and an excellent correlation is observed between the two variables. The following remarks are appropriate here:

(1) The product of the coefficients of variation of the film height and bulk velocity increases as the magnitude of the unsteady term normalized by the mean flow rate increases. This comes as no surprise, as the magnitude of the covariance of the film-height and bulk-velocity fluctuations is expected to be higher in wavier flows (i.e., in flows with increased $\sigma_{\mathrm{h}} / \bar{h}$ ).

(2) Consequently, any data extracted near the flow inlet appear near the origin of the plot, while those extracted from far-downstream locations (e.g., at the PLIF/PTV measurement location, $x=0.256 \mathrm{~m}$ ) are furthest away. 


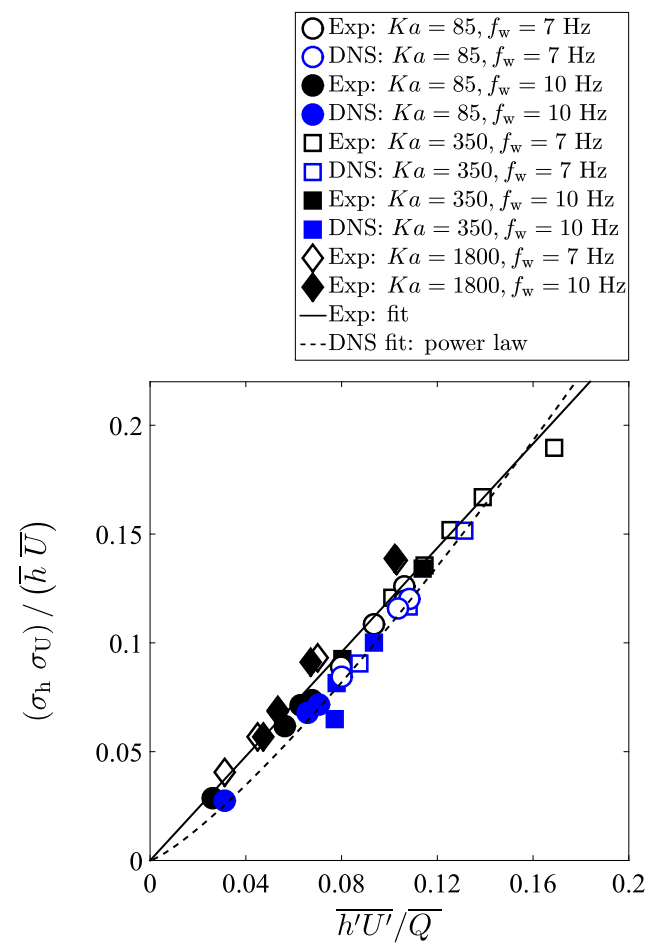

FIG. 10. Product of the coefficients of variation of the film height and bulk velocity, $\sigma_{\mathrm{h}} \sigma_{\mathrm{U}} /(\bar{h} \bar{U})$, against the normalized unsteady term, $\overline{U^{\prime} h^{\prime}} / \bar{Q}$, compiled from both experiments and simulations over all flow conditions in Table I.

(3) The term $y=\sigma_{\mathrm{h}} \sigma_{\mathrm{U}} /(\bar{h} \bar{U})$ can be described as varying as a power law $y=$ a $x^{\mathrm{b}}$ relative to $x=\overline{U^{\prime} h^{\prime}} / \bar{Q}$. At low values of $\sigma_{\mathrm{h}} \sigma_{\mathrm{U}} /(\bar{h} \bar{U}), \sigma_{\mathrm{h}} \sigma_{\mathrm{U}} /(\bar{h} \bar{U}) \leqslant \overline{U^{\prime} h^{\prime}} / \bar{Q}$ (data extracted from near the flow inlet), whereas at higher values $(>0.05)$ this trend is reversed, i.e., $\sigma_{\mathrm{h}} \sigma_{\mathrm{U}} /(\bar{h} \bar{U})>\overline{U^{\prime} h^{\prime}} / \bar{Q}$. However, $\bar{h} \bar{U}<\bar{Q}$ for an unsteady flow, and $\sigma_{\mathrm{h}} \sigma_{\mathrm{U}} \geqslant \overline{U^{\prime} h^{\prime}}$ according to the Cauchy-Schwarz inequality. Consequently, the relation

$$
\frac{\sigma_{\mathrm{h}} \sigma_{\mathrm{U}}}{\bar{h} \bar{U}} \geqslant \frac{\overline{U^{\prime} h^{\prime}}}{\bar{Q}}
$$

must be obeyed, and therefore the relation $\sigma_{\mathrm{h}} \sigma_{\mathrm{U}} /(\bar{h} \bar{U})<\overline{U^{\prime} h^{\prime}} / \bar{Q}$ observed near the origin is most likely due to numerical errors. In addition, when $\overline{U^{\prime} h^{\prime}}=0$ (steady flow), $\sigma_{\mathrm{h}}=0$ and $\sigma_{\mathrm{U}}=0$, and any fit to the data should include the origin.

The same plot of $\sigma_{\mathrm{h}} \sigma_{\mathrm{U}} /(\bar{h} \bar{U})$ as a function of $\overline{U^{\prime} h^{\prime}} / \bar{Q}$ is reproduced in Fig. 10, this time also including experimental results from all flow conditions given in Table I. The experimental data extend to higher $\overline{U^{\prime} h^{\prime}} / \bar{Q}$ values owing to the stronger interface-height and velocity-field fluctuations (discussed earlier in the paper). Specifically, the best (least squares) linear fit to the experimental data in Fig. 10 is described by the relation

$$
\sigma_{\mathrm{h}} \sigma_{\mathrm{U}} /(\bar{h} \bar{U})=1.20 \overline{U^{\prime} h^{\prime}} / \bar{Q},
$$

with a coefficient of determination of $R^{2}=0.96$, whereas for the DNS data we obtain

$$
\sigma_{\mathrm{h}} \sigma_{\mathrm{U}} /(\bar{h} \bar{U})=1.07 \overline{U^{\prime} h^{\prime}} / \bar{Q},
$$

with $R^{2}=0.95$. 
If a power-law fit is employed instead, the relation

$$
\sigma_{\mathrm{h}} \sigma_{\mathrm{U}} /(\bar{h} \bar{U})=1.87\left(\overline{U^{\prime} h^{\prime}} / \bar{Q}\right)^{1.24}
$$

is obtained for the DNS data, with $R^{2}=0.98$. Both the linear and power-law fits are accurate descriptions of the data.

Based on the so-far conducted analysis, the following scenario for the evolution of film unsteadiness is proposed. For a Nusselt flow, for a low-Re flow of very viscous fluid or for the flow immediately downstream of the inlet, no interfacial waves are observed $\left(\sigma_{\mathrm{h}} / \bar{h}=0, \sigma_{\mathrm{U}} / \bar{U}=0\right)$ and the flow is steady. Thus, the film height, bulk velocity, and flow rate are constant, such that $h=\bar{h}=h_{\mathrm{N}}, U=\bar{U}=U_{\mathrm{N}}$, and $Q=\bar{Q}=\bar{h} \bar{U}=h_{\mathrm{N}} U_{\mathrm{N}}$ with $\overline{U^{\prime} h^{\prime}}=0$. The emergence of interfacial waves implies that $\sigma_{\mathrm{h}} / \bar{h}>0$ and $\sigma_{\mathrm{U}} / \bar{U}>0$ as $h, U$, and $Q$ begin to vary spatiotemporally. Consequently, $Q \neq \bar{Q}$ and instead $Q=\bar{Q}+Q^{\prime}=h U$, and $\bar{Q}=\bar{U} \bar{h}+\overline{U^{\prime} h^{\prime}}$. With increasing distance from the inlet, the wave amplitudes grow, resulting in increasing values of $\sigma_{\mathrm{h}} / \bar{h}$ and $\sigma_{\mathrm{U}} / \bar{U}$ until wave growth saturates. Over this development length, $\bar{U} \bar{h} / \bar{Q}$ decrease and $\overline{U^{\prime} h^{\prime}} / \bar{Q}$ increases to compensate.

Throughout the wave development (onset, amplification) process, the film-height and bulkvelocity variations, $\sigma_{\mathrm{h}} / \bar{h}$ and $\sigma_{\mathrm{U}} / \bar{U}$ respectively, are linked to the growth of the unsteady term, $\overline{U^{\prime} h^{\prime}} / \bar{Q}$, and decay of the steady term, $\bar{U} \bar{h} / \bar{Q}$, via a simple relationship. According to the experimental measurements, this relationship is of the form $\sigma_{\mathrm{h}} \sigma_{\mathrm{U}} /(\bar{h} \bar{U})=A_{\mathrm{e}}\left(\overline{U^{\prime} h^{\prime}} / \bar{Q}\right)=$ $A_{\mathrm{e}}(1-\bar{U} \bar{h} / \bar{Q})$ (where $A_{\mathrm{e}}$ is a constant), which is bounded by $\sigma_{\mathrm{h}} \sigma_{\mathrm{U}} /(\bar{h} \bar{U}) \geqslant \overline{U^{\prime} h^{\prime}} / \bar{Q}$, or $A_{\mathrm{e}} \geqslant 1$. According to the simulations, the same relationship is better described by a function of the form $\sigma_{\mathrm{h}} \sigma_{\mathrm{U}} /(\bar{h} \bar{U})=A_{\mathrm{s}}\left(\overline{U^{\prime} h^{\prime}} / \bar{Q}\right)^{B_{\mathrm{s}}}=A_{\mathrm{s}}(1-\bar{U} \bar{h} / \bar{Q})^{B_{\mathrm{s}}}$ (where $A_{\mathrm{s}}$ and $B_{\mathrm{s}}$ are constants). Based on the results presented in Fig. $10, A_{\mathrm{e}}=1.20, A_{\mathrm{s}}=1.87$, and $B_{\mathrm{s}}=1.24$. In finding these constants, data spanning the range $\overline{U^{\prime} h^{\prime}} / \bar{Q} \approx 0.03-0.16$ and $\sigma_{\mathrm{h}} \sigma_{\mathrm{U}} /(\bar{h} \bar{U}) \approx 0.03-0.18$ and stemming from flows covering the range of conditions $\mathrm{Ka}=85,350$, and $1800, \mathrm{Re} \approx 10-290$, and $f_{\mathrm{w}}=7$ and $10 \mathrm{~Hz}$ were used.

From a practical point of view, this is an extremely valuable result; it can be used as a vehicle to link the film-height variations to those of the bulk velocity, irrespective of the flow condition and location relative to the inlet. In the upcoming section, this result will be complemented by a second relationship that will link this analysis with a (quasi)steady analysis based on the Nusselt solution.

At this point, it is essential to discuss further the physical motivation for the linear and power-law fits to the $\sigma_{\mathrm{h}} \sigma_{\mathrm{U}} /(\bar{h} \bar{U})$ and $\overline{U^{\prime} h^{\prime}} / \bar{Q}$ data. Regarding the numerical results of Fig. 9(b), we observe that a power-law fit describes the data better than a linear fit does; however, we also highlight the violation of the Cauchy-Schwartz inequality at lower $\sigma_{\mathrm{h}} \sigma_{\mathrm{U}} /(\bar{h} \bar{U})$ or $\overline{U^{\prime} h^{\prime}} / \bar{Q}$ values. In contrast, the experimental data extracted from $x=0.256 \mathrm{~m}$ (see Fig. 10) suggest a linear relationship without the need to force the fit through the origin. The slope of the fitted line corresponds to $\approx 1.20$ in this case, which provides us with a correlation coefficient between (the instantaneous values of) $h$ and $U$ of $0.83 \bar{Q} /(\bar{h} \bar{U})$. Given that the steady term, $\bar{h} \bar{U} / \bar{Q}$, varies in the range $\approx 0.8-1$ in the experiments depending on the waviness of the flow (lower when the interface is more agitated and vice versa), the correlation coefficient will vary in the range $0.8-1$. Thus, the quasilinear trend between $\sigma_{\mathrm{h}} \sigma_{\mathrm{U}} /(\bar{h} \bar{U})$ and $\overline{U^{\prime} h^{\prime}} / \bar{Q}$ simply highlights the fact that $h$ and $U$ are strongly coupled, irrespective of the film waviness. This forms the basis of the analysis we carried out in Ref. [39], where we linked the instantaneous film height to the instantaneous flow rate via a simple linear relation, as well as the analysis we carry out at present; the accurate prediction of the bulk-flow statistics from the film-height statistics would otherwise be impossible. The fact that, based on the expression we recover by fitting a first-order polynomial to the data, the correlation coefficient falls when the film waviness falls can most probably be attributed to inaccuracies associated with the film-height and bulk-velocity measurements which mainly impact the lower range of measured $\bar{h} \bar{U} / \bar{Q}$ and $\sigma_{\mathrm{h}} \sigma_{\mathrm{U}} /(\bar{h} \bar{U})$. Thus, a power-law fit akin to the one fitted to the numerical data is more representative of the true coupling between $h$ and $U$. 


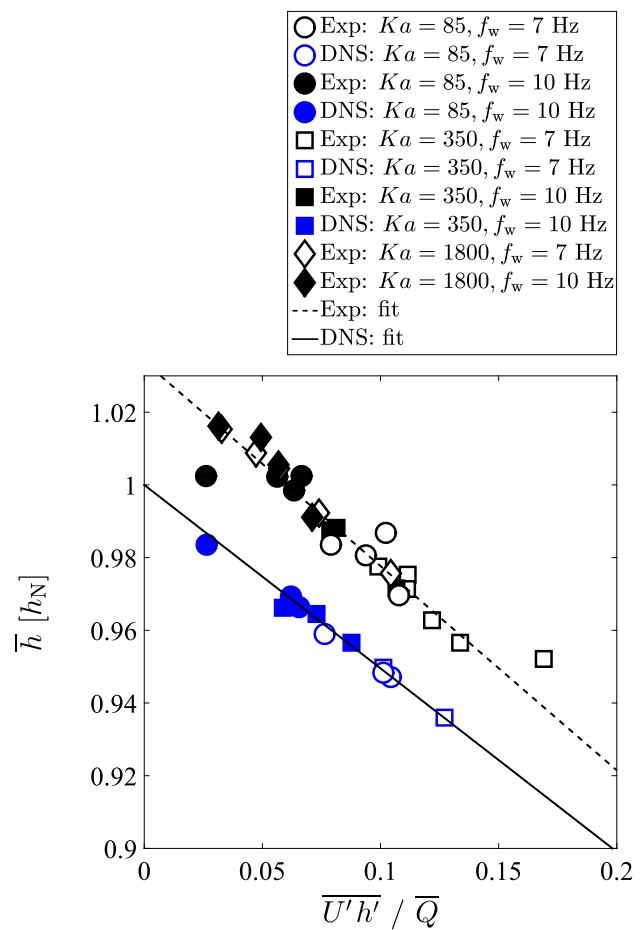

(a)

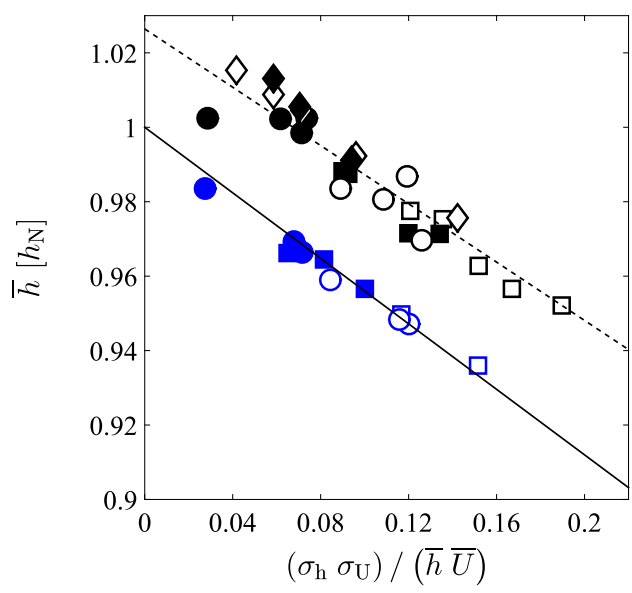

(b)

FIG. 11. Normalized (by the Nusselt height) mean film height $\bar{h} / h_{\mathrm{N}}$, as a function of (a) the unsteady term, $\overline{U^{\prime} h^{\prime}} / \bar{Q}$, and (b) the product of the coefficients of variation of the film height and bulk velocity, $\sigma_{\mathrm{h}} \sigma_{\mathrm{U}} /(\bar{h} \bar{U})$. Linear fits are shown to both experimental and numerical data.

\section{Flow unsteadiness and quasisteady Nusselt flow description}

As already noted in the introduction, despite the fact that the variation of $\bar{h} / h_{\mathrm{N}}$ (ratio of the mean film height to the Nusselt film height) is linked to the interface waviness and associated bulk-velocity fluctuations, a quantitative relation describing this link is not available. In light of our previous analysis, we proceed here to plot $\bar{h} / h_{\mathrm{N}}$ against the corresponding unsteady term, $\overline{U^{\prime} h^{\prime}} / \bar{Q}$, and against the product of the coefficients of variation of the film height and bulk velocity, $\sigma_{\mathrm{h}} \sigma_{\mathrm{U}} /(\bar{h} \bar{U})$, in Fig. 11.

As expected, for the same $\bar{h} / h_{\mathrm{N}}$ values, the experiments show higher film-height and bulkvelocity fluctuations than the corresponding numerical simulations, a result that we associate with the parabolic crestline shapes and increased wave amplitudes along the test-section centerline (see relevant discussion in Sec. IV A). The mean deviation between the measured and numerically obtained mean film heights over all flow conditions with $\mathrm{Ka}=85$ and 350 is $\approx 2.5 \%$, with the latter (simulations) consistently underpredicting the experiments. These deviations may be attributed to small systematic errors in the PLIF measurements (of the order of a few $\mu \mathrm{m}$ ) or the flow-rate and viscosity measurements which are associated with $2 \%$ and $3 \%$ errors respectively, as well as numerical errors. Despite the fact that these deviations are small and the overall agreement between experiments and simulations is very satisfactory, it should be noted that any flow conditions that display $\bar{h} / h_{\mathrm{N}} \geqslant 1$ are mainly related to the $\mathrm{Ka}=1800$ flows in the range $\operatorname{Re}=112-291$, a result that is supported by data provided by Lel et al. [43] $(\mathrm{Re}=2-700)$ and Drosos et al. [46] $(\operatorname{Re}=60-360)$, who have shown that depending on the inclination angle, distance from the inlet, and liquid properties, film flows often obey $\bar{h} \geqslant h_{\mathrm{N}}$ for $\operatorname{Re}$ as low as $\operatorname{Re} \approx 100$. 
A second noteworthy observation concerns the overall trend, which is common to both experiments and simulations, and in particular, the linear decrease of $\bar{h} / h_{\mathrm{N}}$ with both $\overline{U^{\prime} h^{\prime}} / \bar{Q}$ and $\sigma_{\mathrm{h}} \sigma_{\mathrm{U}} /(\bar{h} \bar{U})$ : With this result, we link in a quantitative manner, the Nusselt solution to the NS equation (quasisteady/Nusselt falling-film flow) to the unsteadiness observed in actual wavy films. Both experimental and numerical data are fitted with first-order polynomial functions from the experiments,

$$
\begin{aligned}
& \bar{h} / h_{\mathrm{N}}=-0.56 \overline{U^{\prime} h^{\prime}} / \bar{Q}+1.03\left(\text { with } R^{2}=0.99\right), \\
& \bar{h} / h_{\mathrm{N}}=-0.39 \sigma_{\mathrm{h}} \sigma_{\mathrm{U}} /(\bar{h} \bar{U})+1.03\left(\text { with } R^{2}=0.94\right),
\end{aligned}
$$

and according to the DNSs,

$$
\begin{aligned}
& \bar{h} / h_{\mathrm{N}}=-0.50 \overline{U^{\prime} h^{\prime}} / \bar{Q}+1\left(\text { with } R^{2}=0.98\right), \\
& \bar{h} / h_{\mathrm{N}}=-0.44 \sigma_{\mathrm{h}} \sigma_{\mathrm{U}} /(\bar{h} \bar{U})+1\left(\text { with } R^{2}=0.96\right) .
\end{aligned}
$$

The obtained goodness of fit results are very satisfactory in both experiments and simulations. Furthermore, the gradients from the two methods are closely matched, an interesting result given that in the experiments, data from $\mathrm{Ka}=1800$ flows were also included in deriving these relations (mainly high-Re flows that obey $\bar{h} \geqslant h_{\mathrm{N}}$ ). It should be noted that for flows in the laminar-turbulent transition regime, and more so for values corresponding to fully turbulent flows, a significant deviation from the observed trends is anticipated. It would be important to revisit this remark in further future work, by considering a series of experiments and/or simulations dedicated to addressing the effect of the onset of turbulence on the relations we propose in the present study.

We noted in the introduction that the observation of $\bar{h} / h_{\mathrm{N}} \leqslant 1$ in laminar, wavy film flows has been attributed to the passage of large-amplitude solitary waves and the preceding capillary ripples, which reduce the mean wall shear stress in comparison to a flat film (i.e., when the gas-liquid interface remains undeformed). Here, we observe that in the presence of increased waviness (i.e., for higher values of $\sigma_{\mathrm{h}} \sigma_{\mathrm{U}} /(\bar{h} \bar{U}), \overline{U^{\prime} h^{\prime}} / \bar{Q}$, or $\sigma_{\mathrm{h}}^{2}$ ) and for a fixed $\mathrm{Ka}, \bar{h} / h_{\mathrm{N}}$ falls in a linear manner with any of the three measures of waviness we have discussed so far, at least over the range of attained $\bar{h} / h_{\mathrm{N}}$. Interestingly, in Ref. [41], the intensity of the shear-stress fluctuations was shown to peak at $\operatorname{Re} \approx 35$ for film flows with Ka $=1480$. In our experiments, we observe the lowest $\bar{h} / h_{\mathrm{N}}$ values at $\operatorname{Re} \approx 20$ when $\mathrm{Ka}=85$, and at $\mathrm{Re} \approx 30$ when $\mathrm{Ka}=350$ (see Ref. [38]), which is congruent with the work by Tihon et al. [41]. Furthermore, the variation of $\bar{h} / h_{\mathrm{N}}$ with the flow Re for film flows with $\mathrm{Ka}=85$ and $\mathrm{Ka}=350$ (i.e., for the cases where a clear minimum in $\bar{h} / h_{\mathrm{N}}$ is observed) displays a very similar trend to the variation of the minimum wall shear stress as a function of the flow $\mathrm{Re}$, and an inverse trend to the variation of the maximum wall shear stress as a function of the flow Re, which constitutes further evidence that this is indeed the mechanism that drives the $\bar{h} / h_{\mathrm{N}}$ toward lower values when the film waviness increases. In greater detail, $\bar{h} / h_{\mathrm{N}}$ starts off at $\bar{h} / h_{\mathrm{N}} \approx 1$ at very low Re where the amplitude of the solitary waves is still small, then falls rapidly, reaching a minimum, and finally increases again as the growth of the solitary-wave amplitude saturates.

\section{A METHODOLOGY FOR PREDICTING BULK-VELOCITY STATISTICS}

A methodology for predicting the mean bulk velocity, $\bar{U}$, and bulk-velocity standard deviation, $\sigma_{\mathrm{U}}$, and by extension the steady and unsteady terms, $\bar{U} \bar{h}$ and $\overline{U^{\prime} h^{\prime}}$, in laminar falling-film flows has been developed and will be presented in this section. In the context of a film-flow experiment, we propose that the use of two simple relations, which require information only on the mean flow rate (which can be obtained easily using a flow meter) and the statistics of the film height (specifically only $\bar{h}$ and $\sigma_{\mathrm{h}}$ ) suffices for the recovery of accurate bulk-velocity statistics. An error estimation and quality assessment of the proposed methodology is undertaken separately for the experiments and simulations based on our results, since flow three dimensionality, e.g., due to the presence of side walls in the experiment, can lead to slightly different statistics compared to the simulated infinitely wide 2-D film flows. 
The procedure according to which $\bar{U}$ and $\sigma_{\mathrm{U}}$ are predicted is initiated by considering the correlation between $\bar{h} / h_{\mathrm{N}}$ and $\overline{U^{\prime} h^{\prime}} / \bar{Q}$. In the case of the experiments, this correlation is described by Eq. (27) (repeated from above for convenience),

$$
\bar{h} / h_{\mathrm{N}}=-0.56 \frac{\overline{U^{\prime} h^{\prime}}}{\bar{Q}}+1.03,
$$

while for the DNSs, the same correlation follows Eq. (28),

$$
\bar{h} / h_{\mathrm{N}}=-0.50 \frac{\overline{U^{\prime} h^{\prime}}}{\bar{Q}}+1 .
$$

Using these equations and a knowledge of $\bar{h} / h_{\mathrm{N}}$ and $\bar{Q}$, the magnitude of the unsteady term, $\overline{U^{\prime} h^{\prime}}$, and therefore the steady term, $\bar{U} \bar{h}$, can also be obtained, the latter using

$$
\bar{U} \bar{h}=Q-\overline{U^{\prime} h^{\prime}} .
$$

Based on this result and the direct measurement of $\bar{h}, \bar{U}$ can then be calculated. Finally, using Eqs. (30) and (31), which provide a link between the the unsteady term and the product $\sigma_{\mathrm{h}} \sigma_{\mathrm{U}} /(\bar{h} \bar{U})$, and given our knowledge of the measured $\sigma_{\mathrm{h}}$, one can obtain $\sigma_{\mathrm{U}}$. In the case of the experiments, this final relation is given by Eq. (30) (repeated from above),

$$
\frac{\sigma_{\mathrm{h}} \sigma_{\mathrm{U}}}{\bar{h} \bar{U}}=1.20 \frac{\overline{U^{\prime} h^{\prime}}}{\bar{Q}},
$$

whereas for the DNSs it is given by Eq. (31):

$$
\frac{\sigma_{\mathrm{h}} \sigma_{\mathrm{U}}}{\bar{h} \bar{U}}=1.87\left(\frac{\overline{U^{\prime} h^{\prime}}}{\bar{Q}}\right)^{1.24} .
$$

\section{A. Error estimation}

To assess the efficacy of the proposed methodology and to obtain quantitative estimates of the errors associated with the predictions of $\bar{U}$ and $\sigma_{\mathrm{U}}$, bulk-velocity statistics were estimated using the above relations given the knowledge of the mean and standard deviation of the film-height and mean flow-rate data relating to all flow conditions provided in Table I, which are used as inputs to the relations.

The average absolute deviation (AAD) between the predicted and directly measured or simulated bulk velocities, $\bar{U}$, amounts to $1.3 \%$ and $<1 \%$ respectively, over all flow conditions, with worst-case deviations of $\approx 4 \%$ in both cases. By comparison, the mean deviation between all measured $\bar{U}$ values and those calculated using Eq. (4), the Nusselt expression for the mean bulk velocity, corresponds to $\approx 10 \%$ for the experiments and to $\approx 5 \%$ for the simulations (for flows with $\mathrm{Ka}=85$ and 350 only).

Turning to the predictions of $\sigma_{\mathrm{U}}$, the AAD between measured and predicted values is $8 \%$ for the experiments and $6 \%$ for the simulations. It should be noted that in estimating $\sigma_{\mathrm{U}}$ using Eqs. (30) and (31), we employed the predicted $\bar{U}$ values and not the ones obtained directly from the experiments or simulations. When a linear rather than a power-law fit is employed to link the $\sigma_{\mathrm{h}} \sigma_{\mathrm{U}} /(\bar{h} \bar{U})$ to the unsteady terms in the analysis performed using the DNS data, the mean deviation between the original and predicted values of $\sigma_{\mathrm{U}}$ increased to $8 \%$, still a very satisfactory result.

It is evident then that the accuracy of the proposed methodology in predicting the bulk-velocity statistics of laminar falling films is excellent, at least over the range of flow conditions that were employed toward deriving the relevant relations. We would like to emphasize that in the case of the experiments, the obtained low-deviation values exceeded our expectations, given that the combined PLIF/PTV technique was not optimized for a particular range of flow conditions but was instead developed so that it could be applied over a wide range of flows with reasonable accuracy; the mean film height, for example, varies by a factor of $\approx 4$ across the range of film-flow conditions examined 
TABLE II. Flow conditions and liquid properties of the film flows used to develop the relations provided in Eqs. (32) and (33).

\begin{tabular}{lccrccc}
\hline \hline$\beta[\mathrm{deg}]$ & $f_{\mathrm{w}}[\mathrm{Hz}]$ & $\mathrm{Re}$ & $\mathrm{Ka}$ & $v_{\mathrm{f}}\left[10^{6} \mathrm{~m}^{2} \mathrm{~s}^{-1}\right]$ & $\rho_{\mathrm{f}}\left[\mathrm{kg} \mathrm{m}^{-3}\right]$ & $\gamma_{\mathrm{f}}\left[10^{3} \mathrm{~N} \mathrm{~m}^{-1}\right]$ \\
\hline 20 & 7,10 & $11,21,25$ & 85 & 15.6 & 1170 & 58.7 \\
20 & 7,10 & $45,65,76$ & 350 & 5.77 & 1113 & 59.7 \\
45 & 20 & $60,100,120$ & 4363 & 0.899 & 998 & 72.0 \\
75 & 20 & $40,60,80,100$ & 3932 & 0.899 & 998 & 72.0 \\
90 & 20 & $50,70,100,120$ & 3887 & 0.899 & 998 & 72.0 \\
\hline \hline
\end{tabular}

in this study. Focusing on a narrower range of flows and utilizing higher magnification optics for the velocity measurements could allow a much improved accuracy to be obtained.

A notable limitation associated with the prediction of the mean and standard deviation of the bulk velocity by employment of the above method is the relatively modest sensitivity of $\bar{h} / h_{\mathrm{N}}$ to changes in the product $\bar{h} \bar{U}$ (or equivalently the unsteady flow-rate term, $\overline{U^{\prime} h^{\prime}}$ ). Even though the ensuing effect on the relative error associated with the prediction of $\bar{U}$ is not particularly significant (given that the magnitude of the error in the prediction of $\bar{U}$ is less than $2 \%$ for both experiments and simulations), the error induced in the prediction of $\sigma_{\mathrm{U}}$, both via the inherent uncertainty associated with the correlations (i.e., the fit quality) and the propagation of the uncertainty in $\bar{U}$, can be high. Moreover, when the flow displays limited waviness (i.e., when $\sigma_{\mathrm{h}}$ is small), the relative error in the calculation of $\sigma_{\mathrm{U}}$ can be large. On the other hand, the sensitivity of $\sigma_{\mathrm{h}} \sigma_{\mathrm{U}} /(\bar{h} \bar{U})$ to changes in $\overline{U^{\prime} h^{\prime}}$ is relatively high, which mitigates the aforementioned limitations and is the primary reason for choosing Eqs. (30) and (31) to obtain an estimate of $\sigma_{\mathrm{U}}$, rather than using a relation derived from Fig. 11 (another reason being the higher fit quality obtained in the former). In the next section, we revisit the relations we have used so far to predict $\bar{U}$ and $\sigma_{\mathrm{U}}$, by including a wider range of flow conditions by DNSs of water flows that were not investigated experimentally. This extended study aims to establish the widespread applicability of these relationships in laminar falling-film flows.

\section{B. Extended DNSs}

Here we expand our analysis to include a wider range of flow conditions by DNS as shown in Table II. In addition to the flows with $\mathrm{Ka}=85$ and $\mathrm{Ka}=350\left(\beta=20^{\circ}\right)$ examined earlier, water film flows flowing down $\beta=45^{\circ}, 75^{\circ}$, and finally $90^{\circ}$ (vertical) inclined substrates have been included. The objective behind the inclusion of additional flow conditions is precisely to assess the suitability and generality of the proposed methodology and the validity of our previous analysis over a broader range of flow conditions.

Plots of $\bar{h} / h_{\mathrm{N}}$ against $\overline{U^{\prime} h^{\prime}} / \bar{Q}$ and $\sigma_{\mathrm{h}} \sigma_{\mathrm{U}} /(\bar{h} \bar{U})$ are presented in Fig. 12, while a plot of $\overline{U^{\prime} h^{\prime}} / \bar{Q}$ against $\sigma_{\mathrm{h}} \sigma_{\mathrm{U}} /(\bar{h} \bar{U})$ is presented in Fig. 13. Linear fits are shown in Fig. 12, and a power-law curve fit is shown in Fig. 13.

The relationships that can be used to recover the unsteady term, and by extension the mean and standard deviation of the bulk velocity, are given in Eqs. (32) and (33):

$$
\begin{aligned}
& \bar{h} / h_{\mathrm{N}}=-0.50 \frac{\overline{U^{\prime} h^{\prime}}}{\bar{Q}}+1, \\
& \frac{\sigma_{\mathrm{h}} \sigma_{\mathrm{U}}}{\bar{h} \bar{U}}=1.82\left(\frac{\overline{U^{\prime} h^{\prime}}}{\bar{Q}}\right)^{1.19} .
\end{aligned}
$$

The $R^{2}$ values are 0.98 and $\gg 0.99$, respectively.

Aside from the power-law function fitted to the data in Fig. 13, a linear best fit is also included, with the $y$ intercept not forced through the origin $(0,0)$. The value of $R^{2}$ in this case was better 


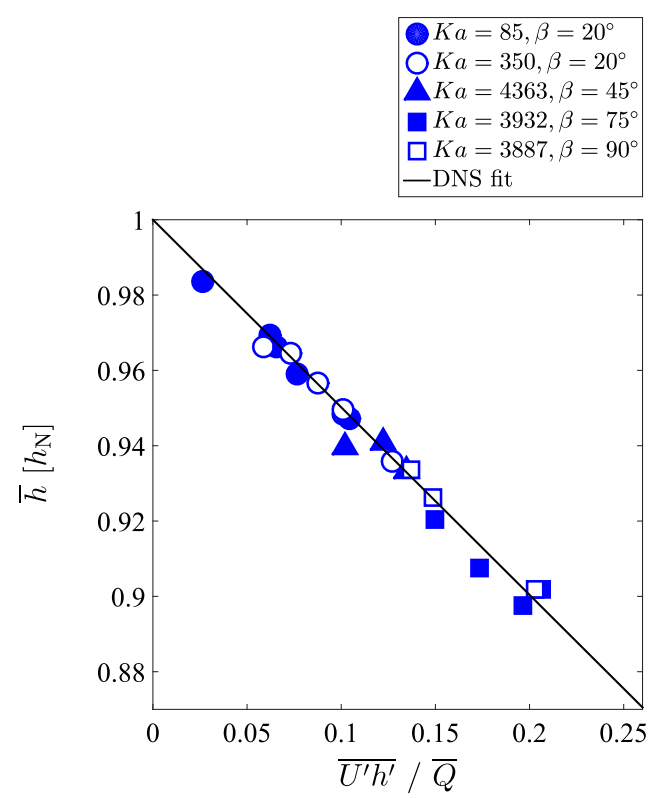

(a)

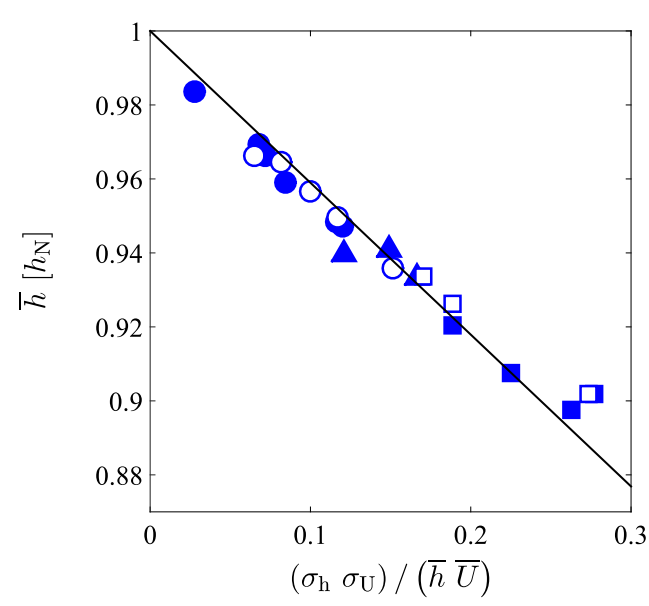

(b)

FIG. 12. Variation of $\bar{h} / h_{\mathrm{N}}$ as a function of (a) $\overline{U^{\prime} h^{\prime}} / \bar{Q}$, and (b) $\sigma_{\mathrm{h}} \sigma_{\mathrm{U}} /(\bar{h} \bar{U})$ obtained by DNS of flows with $\mathrm{Re}, \mathrm{Ka}, \beta$, and $f_{\mathrm{w}}$ as outlined in Table II.

than 0.99, suggesting that based on DNSs, the overall trend is close to linear over the examined range of $\overline{U^{\prime} h^{\prime}} / \bar{Q}$ and $\sigma_{\mathrm{h}} \sigma_{\mathrm{U}} /(\bar{h} \bar{U})$, with the exception of flow conditions with $\overline{U^{\prime} h^{\prime}} / \bar{Q} \leqslant 0.05$ and $\sigma_{\mathrm{h}} \sigma_{\mathrm{U}} /(\bar{h} \bar{U}) \leqslant 0.05$. This particular region of the plot is occupied by data stemming from flows that display very limited waviness, for example, very viscous flows or near-inlet flow data (see, for example, Fig. 9) and are expected to have larger deviations.

As per our previous analysis, aimed at assessing the strength of the proposed methodology and at obtaining quantitative estimates of the errors associated with the predictions of $\bar{U}$ and $\sigma_{\mathrm{U}}$, predictions of $\bar{U}$ and $\sigma_{\mathrm{U}}$ were obtained using the relations in Eqs. (32) and (33), along with the known mean film height, film-height standard deviation, and the mean flow rate for the conditions provided in Table II. The AAD between the predicted and simulated values of $\bar{U}$ is $<1 \%$, while that associated with the prediction of $\sigma_{\mathrm{U}}$ is $\approx 5 \%$. The maximum worst-case deviation is $<20 \%$. It should be noted that we also calculated the deviations over all flow conditions pertaining to the $\mathrm{Ka}=85$ and 350 data sets (i.e., the data sets that were examined earlier, alongside the experimental results, in the previous section) using the above relations rather than those presented in Eqs. (28) and (31) and obtained near-identical values.

\section{Methodology validation}

In this final section we assess the accuracy of our methodology by contrasting results obtained using our proposed relations with experimental and numerical results from flows that were not included in deriving these relations. The relevant flow conditions are provided in Table III. A total of six flow conditions, two from each set of experiments with $\mathrm{Ka}=85,350$, and 1800, were included ( $\beta=20^{\circ}$ in all cases), along with simulations of three flows with (i) $\beta=60^{\circ}, \mathrm{Re}=$ $120, \mathrm{Ka}=4078, f_{\mathrm{w}}=20 \mathrm{~Hz}$; (ii) $\beta=90^{\circ}, \mathrm{Re}=50, \mathrm{Ka}=3887, f_{\mathrm{w}}=50 \mathrm{~Hz}$; and (iii) $\beta=90^{\circ}$, $\mathrm{Re}=70, \mathrm{Ka}=3887, f_{\mathrm{w}}=20 \mathrm{~Hz}$. The distance downstream of the inlet where data were extracted corresponds to $x=256 \mathrm{~mm}$ in the experiments and $x=250 h_{\mathrm{N}}$ in the simulations. 


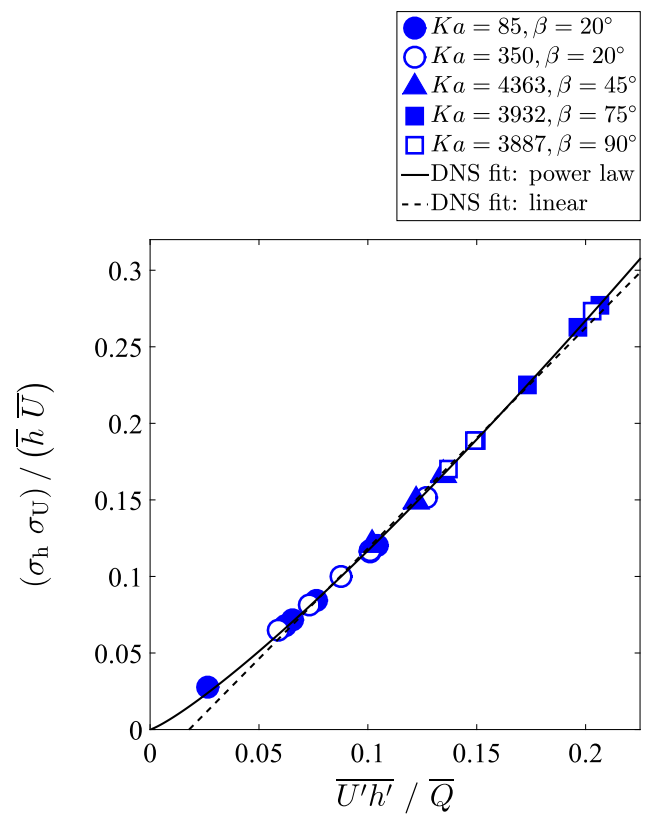

FIG. 13. Plot of the product of the coefficients of variation of the film height and bulk velocity, $\sigma_{\mathrm{h}} \sigma_{\mathrm{U}} /(\bar{h} \bar{U})$, against the relative unsteady terms, $\overline{U^{\prime} h^{\prime}} / \bar{Q}$, compiled using numerical data pertaining to all flow conditions of Table II.

The predicted mean bulk-velocity, $\bar{U}^{*}$, and bulk-velocity standard deviation values, $\sigma_{\mathrm{U}}{ }^{*}$, obtained using Eqs. (27) and (30) in the case of the experiments, and Eqs. (32) and (33) in the simulations, were normalized by the experimental and numerical results respectively and are presented in Fig. 14 as a function of the flow Re. The mean and maximum relative deviations between $\bar{U}^{*}$ and $\bar{U}$ over the six experimentally investigated flow conditions in the validation exercise amount to $2.2 \%$ and $5 \%$ respectively, while for the three flow conditions examined by simulations, both mean and maximum relative deviations amount to $<1 \%$. Further, the mean and maximum relative deviations between $\sigma_{\mathrm{U}}{ }^{*}$ and $\sigma_{\mathrm{U}}$ amount to $6 \%$ and $11 \%$ in the experiments and $4 \%$ and $8 \%$ in the simulations. Hence, in both experiments and simulations, the observed deviations do not exceed those obtained earlier between the experimental and numerical data employed to develop the relations and the predicted values of $\bar{U}$ and $\sigma_{\mathrm{U}}$ based on these relations.

TABLE III. Flow conditions and liquid properties of the film flows used to assess the validity of the experimentally derived correlations provided in Eqs. (27) and (30), and the DNS-based correlations provided in Eqs. (32) and (33).

\begin{tabular}{lccrcccc}
\hline \hline$\beta[\mathrm{deg}]$ & $f_{\mathrm{w}}[\mathrm{Hz}]$ & $\mathrm{Re}$ & $\mathrm{Ka}$ & $\nu_{\mathrm{f}}\left[10^{6} \mathrm{~m}^{2} \mathrm{~s}^{-1}\right]$ & $\rho_{\mathrm{f}}\left[\mathrm{kg} \mathrm{m}^{-3}\right]$ & $\gamma_{\mathrm{f}}\left[10^{3} \mathrm{~N} \mathrm{~m}^{-1}\right]$ & Method \\
\hline 20 & 10 & 12,29 & 85 & 15.6 & 1170 & 58.7 & Exp \\
20 & 10 & 45,77 & 350 & 5.77 & 1113 & 59.7 & Exp \\
20 & 7 & 175,257 & 1800 & 1.71 & 1054 & 58.1 & Exp \\
60 & 20 & 120 & 4078 & 0.899 & 998 & 72.0 & DNS \\
90 & 50 & 50 & 3887 & 0.899 & 998 & 72.0 & DNS \\
90 & 20 & 70 & 3887 & 0.899 & 998 & 72.0 & DNS \\
\hline \hline
\end{tabular}




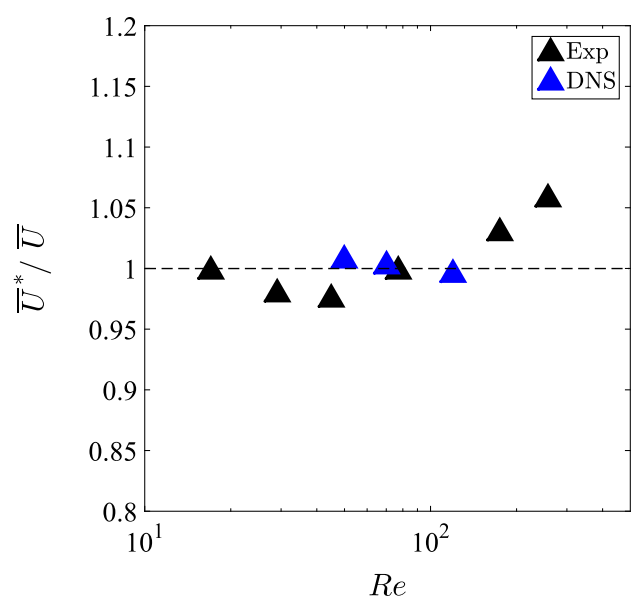

(a)

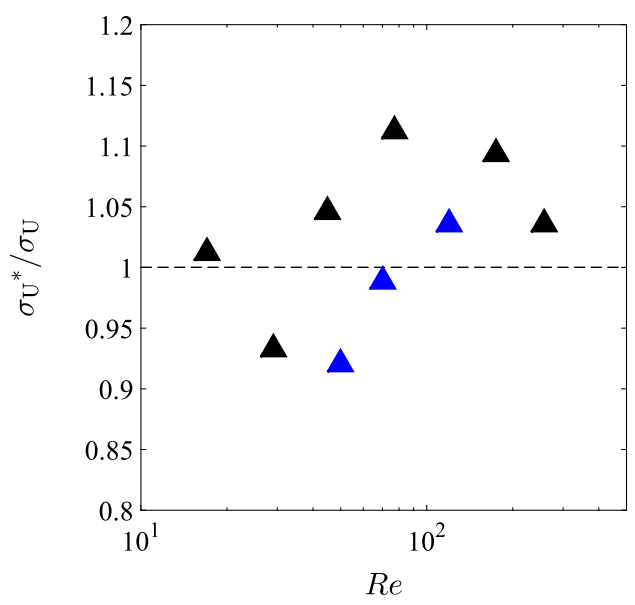

(b)

FIG. 14. Predicted values of the bulk velocity: (a) mean, $\bar{U}^{*}$, and (b) standard deviation, $\sigma_{\mathrm{U}}{ }^{*}$, normalized by direct experimental and numerical results from the same flow conditions (in Table III) and plotted as a function of the flow.

\section{CONCLUSIONS}

An optical measurement technique that combines simultaneous planar laser-induced fluorescence (PLIF) with particle tracking velocimetry (PTV) was employed alongside direct numerical simulations (DNSs) for the detailed characterization of the flow and interface statistics of harmonically excited, planar falling-film flows. PLIF was employed in order to measure spaceand time-resolved film thickness, and PTC was employed to obtain 2-D velocity-field measurements underneath the wavy film interface. The experiments used three different aqueous-glycerol solutions corresponding to Kapitza numbers $\mathrm{Ka}=85,350$, and 1800, spanned the range of Reynolds numbers $\mathrm{Re}=10-291$, and had two inlet forcing frequencies $f_{\mathrm{w}}=7$ and $10 \mathrm{~Hz}$. Based on the PLIF and PTV data, the variations of film height, bulk velocity, and flow rate were recovered along the wave topology. The uncertainties associated with the film-height and bulk-velocity measurements are estimated at $\leqslant 3 \%$ and $\approx 3 \%$, respectively, while the error quoted for the PLIF- and PTV-derived mean flow-rate measurements corresponds to $3 \%$.

Building upon our previous research effort in Ref. [1], we generated film-height, bulk-velocity, and flow-rate time series in order to study the dynamics of the unsteady film flows, and in particular the film-height and bulk-velocity fluctuations over the range of experimentally and numerically investigated flow conditions. In doing so, the local and instantaneous flow rate was decomposed into steady and unsteady terms, $\bar{U} \bar{h}$ and $\overline{U^{\prime} h^{\prime}}$, respectively, which were compared to the filmheight and bulk-velocity coefficients of variation, $\sigma_{\mathrm{h}} / \bar{h}$ and $\sigma_{\mathrm{U}} / \bar{U}$, respectively, as well as to results from quasisteady Nusselt flows. The most significant results that emerge from this analysis can be summarized below as follows:

(1) The steady flow-rate (mass-transfer) term, $\bar{U} \bar{h}$, varies linearly with the flow Re, and the unsteady flow-rate term, $\overline{U^{\prime} h^{\prime}}$, varies linearly with the film-height variance, $\sigma_{\mathrm{h}}{ }^{2}$, based on both experimental and numerical results, which generally showed excellent agreement.

(2) The product of the coefficients of variation of the film height and bulk velocity, $\sigma_{\mathrm{U}} \sigma_{\mathrm{h}} /(\bar{U} \bar{h})$, increases as the magnitude of the unsteady term relative to the mean flow rate, $\overline{U^{\prime} h^{\prime}} / \bar{Q}$, increases. This comes as no surprise, as the magnitude of the covariance of the film-height and bulk-velocity fluctuations is expected to be higher for a wavier flow (i.e., a flow that displays more pronounced film-height and bulk-velocity fluctuations). 
(3) This trend is observed in both experiments and simulations at a downstream distance of $x=0.256 \mathrm{~m}$, where the PLIF/PTV data were generated, as well as in simulations of the same flows that included data over a range of distances, closer to the flow inlet. It is also found that with increasing distance from the flow inlet, the normalized steady term $\bar{U} \bar{h} / \bar{Q}$ decreases, while the normalized unsteady term $\overline{U^{\prime} h^{\prime}} / \bar{Q}$ increases. Linear fits can be used to describe these trends in both the experiments and simulations; however, a power-law curve is found to fit the numerical data more closely.

(4) The terms $\sigma_{\mathrm{U}} \sigma_{\mathrm{h}} / \bar{U} \bar{h}$ and $\overline{U^{\prime} h^{\prime}} / \bar{Q}$ are both found to decrease linearly with the normalized (by the Nusselt flow) thickness, $\bar{h} / h_{\mathrm{N}}$; a result that links quantitatively the Nusselt solution to the unsteadiness observed in real wavy falling films (and specifically, to the magnitude of these unsteady terms).

(5) Based on the relations that were derived to describe the aforementioned trends, and also the knowledge of the mean film height, $\bar{h}$, film-height standard deviation, $\sigma_{\mathrm{h}}$, and mean flow rate, $\bar{Q}$, a methodology for predicting the mean bulk velocity, $\bar{U}$, and bulk-velocity standard deviation, $\sigma_{\mathrm{U}}$, is presented. In the experiments, the errors associated with the predictions of $\bar{U}$ and $\sigma_{\mathrm{U}}$ are estimated at $\approx 1.5 \%$ and $8 \%$, while in the simulations, these amount to $<1 \%$ and $<2 \%$, respectively.

And as already mentioned, of particular interest would be the effect of the onset of turbulence on the relations we propose here. We hope to examine this and related issues in future studies.

\section{ACKNOWLEDGMENTS}

This work was supported by the UK Engineering and Physical Sciences Research Council (EPSRC) [Grants No. EP/J006041/1, No. EP/K008595/1, No. EP/L020564/1, and No. EP/M021556/1]. We are grateful to Dr. Marc Pradas for numerous stimulating discussions on the nonlinear dynamics of falling film flows.

Data supporting this publication can be obtained on request from cep-lab@imperial.ac.uk.

[1] N. Brauner and D. M. Maron, Characteristics of inclined thin films, waviness, and the associated mass transfer, Int. J. Heat Mass Transf. 25, 99 (1982).

[2] I. Mudawar and R. A. Houpt, Measurement of mass and momentum transport in wavy-laminar falling liquid films, Int. J. Heat Mass Transf. 36, 4151 (1993).

[3] S. V. Alekseenko, V. E. Nakoryakov, and B. G. Pokusaev, Wave effect on the transfer processes in liquid films, Chem. Eng. Commun. 141, 359 (1996).

[4] C. N. Markides, R. Mathie, and A. Charogiannis, An experimental characterization of spatiotemporally resolved heat transfer in thin liquid-film flows falling over an inclined heated foil, Int. J. Heat Mass Transf. 93, 872 (2015).

[5] P. L. Kapitza, Wave flow of thin layers of a viscous fluid: I. Free flow, J. Exp. Theor. Phys. 18, 3 (1948).

[6] P. L. Kapitza and S. P. Kapitza, Wave flow of thin layers of a viscous fluid III: Experimental study of undulatory flow conditions, J. Exp. Theor. Phys. 19, 105 (1949).

[7] C. S. Yih, Stability of liquid flow down an inclined plane, Phys. Fluids 6, 321 (1963).

[8] E. A. Demekhin, E. N. Kalaidin, S. Kalliadasis, and S. Yu. Vlaskin, Three-dimensional localized coherent structures of surface turbulence. I. Scenarios of two-dimensional-three-dimensional transition, Phys. Fluids 19, 114103 (2007).

[9] E. A. Demekhin, E. N. Kalaidin, S. Kalliadasis, and S. Yu. Vlaskin, Three-dimensional localized coherent structures of surface turbulence. II. $\Lambda$ solitons, Phys. Fluids 19, 114104 (2007).

[10] E. A. Demekhin, E. N. Kalaidin, and A. S. Selin, Three-dimensional localized coherent structures of surface turbulence. III. Experiment and model validation, Phys. Fluids 22, 092103 (2010).

[11] E. A. Demekhin, E. N. Kalaidin, S. Kalliadasis, and S. Yu. Vlaskin, Three-dimensional localized coherent structures of surface turbulence: Model validation with experiments and further computations, Phys. Rev. E 82, 036322 (2010). 
[12] S. Kalliadasis, C. Ruyer-Quil, B. Scheid, and M. G. Velarde, Falling Liquid Films, Springer Series on Applied Mathematical Sciences Vol. 176 (Springer Verlag, Berlin, 2012).

[13] B. Scheid, C. Ruyer-Quil, and P. Manneville, Wave patterns in film flows: Modelling and three-dimensional waves, J. Fluid Mech. 562, 183 (2006).

[14] N. Kofman, S. Mergui, and C. Ruyer-Quil, Three-dimensional instabilities of quasi-solitary waves in falling liquid films, J. Fluid Mech. 757, 854 (2014).

[15] M. Rietz, W. Rohlfs, R. Kneer, and B. Scheid, Experimental investigation of thermal structures in regular three-dimensional falling films, Eur. Phys. J. Spec. Top. 224, 355 (2015).

[16] S. V. Alekseenko, V. V. Guzanov, D. M. Markovich, and S. M. Kharlamov, Specific features of a transition from the regular two-dimensional to three-dimensional waves on falling liquid films, Tech. Phys. Lett. 38, 739 (2012).

[17] S. V. Alekseenko, A. V. Bobylev, V. V.G uzanov, D. M. Markovich, and S. M. Kharlamov, Formation of rivulets in isothermal liquid film flow during transition to a three-dimensional wave regime, Tech. Phys. Lett. 40, 1031 (2014).

[18] P. N. Yoshimura, T. Nosoko, and T. Nagata, Enhancement of mass transfer into a falling laminar liquid film by two-dimensional surface waves-Some experimental observations and modelling, Chem. Eng. Sci. 51, 1231 (1996).

[19] S. M. Kharlamov, V. V. Guzanov, A. V. Bobylev, S. V. Alekseenko, and D. M. Markovich, The transition from two-dimensional to three-dimensional waves in falling liquid films: Wave patterns and transverse redistribution of local flow rates, Phys. Fluids 27, 114106 (2015).

[20] J. Liu, J. D. Paul, and J. P. Gollub, Measurements of the primary instabilities of film flows, J. Fluid Mech. 250, 69 (1993).

[21] J. Liu and J. P. Gollub, Onset of Spatially Chaotic Waves on Flowing Films, Phys. Rev. Lett. 70, 2289 (1993).

[22] J. Liu and J. P. Gollub, Solitary wave dynamics of film flows, Phys. Fluids 6, 1702 (1994).

[23] J. Liu, J. B. Schneder, and J. P. Gollub, Three-dimensional instabilities of film flows, Phys. Fluids 7, 55 (1995).

[24] T. Nosoko, P. N. Yoshimura, T. Nagata, and K. Oyakawa, Characteristics of two-dimensional waves on falling liquid films, Chem. Eng. Sci. 51, 725 (1996).

[25] T. Nosoko and A. Miyara, The evolution and subsequent dynamics of waves on a vertically falling liquid film, Phys. Fluids 16, 1118 (2004).

[26] B. Ramaswamy, S. Chippada, and S. W. Joo, A full-scale numerical study of interfacial instabilities in thin-film flows, J. Fluid Mech. 325, 163 (1996).

[27] K. Argyriadi, K. Serifi, and V. Bontozoglou, Nonlinear dynamics of inclined films under low-frequency forcing, Phys. Fluids 16, 2457 (2004).

[28] N. A. Malamataris, M. Vlachogiannis, and V. Bontozoglou, Solitary waves on inclined films: Flow structure and binary interactions, Phys. Fluids 14, 1082 (2002).

[29] Y. Y. Trifonov, Stability of the wavy film falling down a vertical plate: The DNS computations and Floquet theory, Int. J. Multiphase Flow 61, 73 (2014).

[30] D. Reck and N. Aksel, Recirculation areas underneath solitary waves on gravity-driven film flows, Phys. Fluids 27, 112107 (2015).

[31] W. Nusselt, Die oberflachenkondensation des wasserdampfes, Zetrschr. Ver. Deutch. Ing. 60, 541 (1916).

[32] N. A. Malamataris and V. Balakotaiah, Flow structure underneath the large amplitude waves of a vertically falling film, AIChE J. 54, 1725 (2008).

[33] F. Denner, M. Pradas, A. Charogiannis, C. N. Markides, B. G. M. van Wachem, and S. Kalliadasis, Self-similarity of solitary waves on inertia-dominated falling liquid films, Phys. Rev. E 93, 033121 (2016).

[34] G. Karimi and M. Kawaji, An experimental study of freely falling films in a vertical tube, Chem. Eng. Sci. 53, 3501 (1998).

[35] G. Karimi and M. Kawaj, Flooding in vertical counter-current annular flow, Nucl. Eng. Des. 200, 95 (2000).

[36] K. Moran, J. Inumaru, and M. Kawaji, Instantaneous hydrodynamics of a laminar wavy liquid film, Int. J. Multiphase Flow 28, 731 (2002). 
[37] P. Adomeit and U. Renz, Hydrodynamics of three-dimensional waves in laminar falling films, Int. J. Multiphase Flow 26, 1183 (2000).

[38] A. Charogiannis, J. S. An, and C. N. Markides, A simultaneous planar laser-induced fluorescence, particle image velocimetry and particle tracking velocimetry technique for the investigation of thin liquid-film flows, Exp. Therm. Fluid Sci. 68, 516 (2015).

[39] A. Charogiannis, F. Denner, B. G. M. van Wachem, S. Kalliadasis, and C. N. Markides, Detailed hydrodynamic characterization of harmonically excited falling-film flows: A combined experimental and computational study, Phys. Rev. Fluids 2, 014002 (2017).

[40] J. Tihon, V. Tovchigrechko, V. Sobolik, and O. Wein, Electrodiffusion detection of the near-wall flow reversal in liquid films at the regime of solitary waves, J. Appl. Electrochem. 33, 577 (2003).

[41] J. Tihon, K. Serifi, K. Argyriadi, and V. Bontozoglou, Solitary waves on inclined films: Their characteristics and the effects of wall shear stress, Exp. Fluids 41, 79 (2006).

[42] H. Takahama and S. Kato, Longitudinal flow characteristics of vertically falling liquid films without concurrent gas flow, Int. J. Multiphase Flow 6, 203 (1980).

[43] V. V. Lel, F. Al-Sibai, A. Leefken, and U. Renz, Local thickness and wave velocity measurement of wavy films with a chromatic confocal imaging method and a fluorescence intensity technique, Exp. Fluids 39, 856 (2005).

[44] D. W. Zhou, T. Gambaryan-Roisman, and P. Stephan, Measurement of water falling film thickness to flat plate using confocal chromatic sensor technique, Exp. Therm. Fluid Sci. 33, 273 (2009).

[45] W. Ambrosini, N. Forgione, and F. Oriolo, Statistical characteristics of water film falling down a flat plate at different inclinations and temperatures, Int. J. Multiphase Flow 28, 1521 (2002).

[46] E. I. P. Drosos, S. V. Paras, and A. J. Karabelas, Characteristics of developing free falling films at intermediate Reynolds and high Kapitza numbers, Int. J. Multiphase Flow 30, 853 (2004).

[47] T. D. Karapantsios, S. V. Paras, and A. J. Karabelas, Statistical characteristics of free falling films at high Reynolds numbers, Int. J. Multiphase Flow 15, 1 (1989).

[48] I. Zadrazil, O. K. Matar, and C. N. Markides, An experimental characterization of downwards gas-liquid annular flow by laser-induced fluorescence: Flow regimes and film statistics, Int. J. Multiphase Flow 60 , 87 (2014).

[49] F. Denner and B. G. M. van Wachem, Fully-coupled balanced-force VOF framework for arbitrary meshes with least-squares curvature evaluation from volume fractions, Numer. Heat Transfer, Part B 65, 218 (2014).

[50] F. Denner, D. R. van der Heul, G. T. Oud, M. M. Villar, A. S. Neto, and B. G. M. van Wachem, Comparative study of mass-conserving interface capturing frameworks for two-phase flows with surface tension, Int. J. Multiphase Flow 61, 37 (2014).

[51] F. Denner and B. G. M. van Wachem, Compressive VOF method with skewness correction to capture sharp interfaces on arbitrary meshes, J. Comput. Phys. 279, 127 (2014).

[52] F. Denner and B. G. M. van Wachem, On the convolution of fluid properties and surface force for interface capturing methods, Int. J. Multiphase Flow 54, 61 (2013).

[53] F. Denner and B. G. M. van Wachem, Numerical time-step restrictions as a result of capillary waves, J. Comput. Phys. 285, 24 (2015).

[54] C. W. Hirt and B. D. Nichols, Volume of fluid (VOF) method for the dynamics of free boundaries, J. Comput. Phys. 39, 201 (1981).

[55] J. U. Brackbill, D. B. Kothe, and C. Zemach, A continuum method for modeling surface tension, J. Comput. Phys. 100, 335 (1992).

[56] A. Georgantaki, J. Vatteville, M. Vlachogiannis, and V. Bontozoglou, Measurements of liquid film flow as a function of fluid properties and channel width: Evidence for surface-tension-induced long range transverse coherence, Phys. Rev. E 84, 026325 (2011).

[57] M. Vlachogiannis, A. Samandas, V. Leontidis, and V. Bontozoglou, Effect of channel width on the primary instability of inclined film flow, Phys. Fluids 22, 012106 (2010).

[58] V. Leontidis, J. Vatteville, M. Vlachogiannis, N. Andritsos, and V. Bontozoglou, Nominally twodimensional waves in inclined film flow in channels of finite width, Phys. Fluids 22, 112106 (2010). 